NBER WORKING PAPER SERIES

\title{
MOMENTUM CYCLES AND LIMITS TO ARBITRAGE EVIDENCE FROM VICTORIAN ENGLAND AND POST-DEPRESSION US STOCK MARKETS
}

\author{
Benjamin Chabot \\ Eric Ghysels \\ Ravi Jagannathan \\ Working Paper 15591 \\ http://www.nber.org/papers/w15591
}

\author{
NATIONAL BUREAU OF ECONOMIC RESEARCH \\ 1050 Massachusetts Avenue \\ Cambridge, MA 02138
}

December 2009

We thank Nick Barberis, Jennifer Conrad, Gautam Kaul, Jegadeesh Narasimhan, Lu Zhang, and seminar participants at the University of Michigan for helpful comments. We also thank Soohun Kim for invaluable research assistance. The views expressed herein are those of the author(s) and do not necessarily reflect the views of the National Bureau of Economic Research.

NBER working papers are circulated for discussion and comment purposes. They have not been peerreviewed or been subject to the review by the NBER Board of Directors that accompanies official NBER publications.

(C) 2009 by Benjamin Chabot, Eric Ghysels, and Ravi Jagannathan. All rights reserved. Short sections of text, not to exceed two paragraphs, may be quoted without explicit permission provided that full credit, including $\odot$ notice, is given to the source. 
Momentum Cycles and Limits to Arbitrage Evidence from Victorian England and Post-Depression US Stock Markets

Benjamin Chabot, Eric Ghysels, and Ravi Jagannathan

NBER Working Paper No. 15591

December 2009

JEL No. G0,G10,G12,G14

\title{
ABSTRACT
}

We evaluate the importance of "Limits to Arbitrage" to explain profitability of momentum strategies. Specifically, when the availability of arbitrage capital is in short supply, momentum cycles last longer, and breaks in momentum cycles are shorter. We demonstrate the robustness of our findings with a unique database of stock returns from1866-1907 London and the CRSP database. Momentum cycle durations are similar in both databases and all other momentum facts documented in the literature using the CRSP database hold for the Victorian period as well, except for the January reversal due to the absence of capital gains taxation.

\author{
Benjamin Chabot \\ Ravi Jagannathan \\ Department of Economics \\ Kellogg School of Management \\ Yale University \\ 27 Hillhouse Ave, Rm 33 \\ New Haven, CT 06520 \\ Northwestern University \\ 2001 Sheridan Road \\ and NBER \\ benjamin.chabot@yale.edu \\ 431 Jacobs Center \\ Evanston, IL 60208-2001 \\ and NBER \\ Eric Ghysels \\ rjaganna@northwestern.edu \\ Department of Economics \\ University of North Carolina-Chapel Hill \\ Gardner Hall, CB 3305 \\ Chapel Hill, NC 27599-3305 \\ eghysels@unc.edu
}


Momentum strategies - buying winners and selling losers - have generated abnormal returns for over 160 years. We make use of recently collected historical data to document the returns to momentum investing in the CRSP era United States and the Victorian era London Stock Exchange. A consistently applied momentum strategy generated similar abnormal returns and profit cycles across both periods. We suggest an explanation for the both the persistence and cyclical nature of abnormal momentum profits. While buying winners and selling losers consistently generates abnormal returns, the momentum strategy does expose investors to large losses with enough regularity to limit leverage. We show that while the average return to the momentum strategy is high and uncorrelated with the market index, capturing these abnormal returns exposes the momentum investor to occasional sudden losses. We speculate that these losses make it difficult for sophisticated investors to consistently employ leverage with other people's money. This "separation of brains from capital" can explain both the occasional large losses to the momentum strategy apparent in the time series of returns and why momentum continued to be a profitable strategy for 140 years. Sophisticated momentum traders subject to margin or capital constraints can exacerbate losses if forced to unwind positions at the end of profit cycles and this may impose a classic limit to arbitrage.

The paper makes three contributions. First, we document that the momentum strategy has generated abnormal returns for over 140 years - using a new hand-collected data set of the London Stock Exchange during the Victorian era and comparing it with the Post Depression US. Second, we establish the fact that momentum portfolios have been subject to similar cycles since the 1860s. Third, we suggest a new approach to testing the sources of momentum cycles based on the duration dynamics of moment profit cycles. More specifically, we examine return distributions and the duration spells of momentum profit and losses from both eras. We test the underlying null hypothesis that the momentum portfolio returns and duration spells are drawn from the same distributions across eras. What then explains the persistence of momentum? We think the answer lies in the fact that while momentum portfolios do generate high returns orthogonal to market and business cycle risks, the momentum strategy also exposes investors to high variance and frequent losses. The frequent loses associated with momentum strategies make it difficult for investors who use other people's money or leverage to drive momentum profits out of the market. This is the well known "limits to arbitrage" explanation of persistent anomalies. To test this formally, we introduce a new methodology based on modeling 
the duration dynamics as a function of the state of the economy, the market return and the scarcity of capital. If capital constraints limit arbitrage we would expect a measure of capital scarcity to predict the duration of cycles. We find strong evidence supporting this explanation of momentum cycles.

We make use of our long times series of momentum profits to identify states in which the hazard of loss is high. A simple measure of the scarcity of investment capital - the risk-free rate - predicts the duration of momentum profit or loss cycles in both eras. We hypothesize that markets always have noise traders who buy and sell for idiosyncratic liquidity reasons or are subject to the biases in the literature. Profit opportunities from a simple momentum trading rule should depend on the relative supply of capital available to sophisticated and noise traders. Noise traders slow the adjustment of prices to new information and create opportunities for momentum traders. Time periods where investment capital is plentiful are also periods where the market is awash in sophisticated traders who find it easy to attract arbitrage capital and expand their positions. Momentum profits are therefore most likely to persist in periods where capital available to sophisticated momentum traders is in short supply relative to noise traders. This is the familiar story of rational but capital constrained traders. When investment capital is plentiful, the entry of sophisticated leveraged traders can quickly exhaust the profits from trading rules. As profits are exhausted and sophisticated traders post losses with more regularity, sophisticated traders exit and the profit opportunities return.

Any duration based test of a trading strategy requires a large sample of observed cycles. A test based on the availability of arbitrage capital is best evaluated with an evaluation of a long time series rather than a cross-country comparison of momentum cycles. With increasing globalization, arbitrageurs in one country can exploit with ease profitable trading opportunities in other countries. That makes it difficult to form country specific measures of arbitrage capital since capital can easily move across national borders and draws into question whether crosscountry cycles are independent observations. We therefore focus on the largest stock markets and establish the robustness of our findings by examining long time series. We therefore use CRSP era US stock market data and Victorian era London stock market returns to examine our hypothesis.

In the paragraphs to follow, we review the literature and document the facts about risk, return and cyclicality of momentum portfolios during the CRSP and Victorian eras. 


\section{A. Stock Price Momentum during CRSP and Victorian Age: Cyclicality with}

\section{Market States}

We use a new hand-collected data set of the London Stock Exchange. ${ }^{1}$ The new data set consists of the closing prices, dividends and shares outstanding of 1,808 stocks (equity) listed in London between 1866 and 1907. These stocks represent virtually every stock traded on the London Stock Exchange during this period. The fact that the London Stock Exchange was the most important market at the time, and that the U.K. was riding high on the waves of the second industrial revolution makes this a particularly interesting era to study as we cover a period of prosperity, expansion and the harbinger of twentieth century capitalism and financial markets.

To the extent decision making biases are hardwired into the human psyche, we should expect to find price momentum in stocks even during the second industrial revolution. The London market during the Victorian age was, by today's standards, a primitive market with high execution costs, limited liquidity and very elementary computational power to sustain complex trading strategies. Even today's emerging markets may look quite advanced in comparison to $19^{\text {th }}$ century London, at least the know-how of trading and financial theory have made big leaps forward compared to what was available more than 100 years ago. Hence, if we did not find relative price momentum in stocks during the Victorian age, that would for instance cast doubt upon the behavioral explanations that rely on the psychology of decision making.

The existence of price momentum during the Victorian age will not by itself rule out any one class of theories, but does eliminate the possibility that price momentum may be an artifact of data mining. Furthermore, to the extent that existing theories place out of sample restrictions on historical data, we can use our $19^{\text {th }}$ century sample to evaluate competing hypotheses. We find both statistically and economically significant momentum effects short run reversal, medium term continuation, and long run reversal in past winners minus past losers portfolio returns - and the order of magnitude is quite similar to that of the

\footnotetext{
${ }^{1}$ Historical data have been used before to assess some of the salient empirical stylized facts of asset returns. Most of these studies have focused on issues such as long term predictability, see e.g. for NYSE from 1815 to 1925, as discussed in Goetzmann (1993) and Goetzmann, Ibbotson and Peng (2001) or the Brussels stock exchange as discussed in Annaert and Van Hyfte (2006).
} 
widely documented end of $20^{\text {th }}$ century evidence. However, we do not find a particularly strong relationship between momentum profits and firm size and we do not find a distinct January effect in our data. ${ }^{2}$

An important defining characteristic of momentum profits we find is that momentum profits are cyclical. Figure 1 plots the time series of the Fama-French momentum factor returns from January 1946 to May 2008 and the return on a similarly constructed momentum factor during the Victorian era - based on the aforementioned new hand-collected data set described in detail later. When we compare the Post-War data with the Victorian era we observe the same cyclical pattern as well as momentum profits with roughly the same range of monthly gains/losses. The similarities are striking: Post-War momentum profits as measured by the one year moving average of past Winners minus past Losers returns exhibited negative episodes (i.e., vanished) once every 2 years with an average duration of 4.1 months per episode. During the period January 1867 to December 1907, momentum returns exhibited negative episodes once every 1.4 years with an average duration of 3.8 months per episode. ${ }^{3}$ The timeseries of CRSP and historical era momentum profits resembles other rule-based trading strategies subject to the limits of arbitrage (see e.g. Shleifer and Vishny (1997)) - high average returns with enough periodic declines to prevent sophisticated leveraged investors from capturing profits without risk. $^{4}$

We are not the first to notice cyclicality in momentum returns. Cyclicality is consistent with many behavioral explanations of momentum. One that makes testable predictions about observable historical returns is Cooper, Gutierrez and Hameed (2004) (hereafter CGH). CGH note that the theory of Daniel, Hirshleifer, and Subrahmanyam (1997) (henceforth DHS) can be extended to predict differences in momentum profits across states of the market, like bull and bear markets, as aggregate overconfidence should be greater following

\footnotetext{
${ }^{2}$ Grinblatt and Moskowitz (2004) carefully document the importance of tax-loss selling in momentum portfolios. The absence of a January effect in our Victorian data is not surprising as Victorians did not tax capital gains and the Victorian tax year did not end in December.

${ }^{3}$ Griffin, Ji and Martin (2004) have documented similar periodic declines in other nations as well. Figure 1 involves a one-year centered moving average. Hence, the moving average scheme produces some induced temporal dependence. As will be discussed shortly, the temporal dependence in momentum profits goes beyond that induced by smoothing.

${ }^{4}$ Gatev, Goetzmann and Rouwenhorst (2006) note that pairs trading - like momentum trading - features periodic breaks as well. Hence, momentum trading; pairs trading; and the like - i.e. certain rules of forming portfolios and liquidating portfolios - appear to work with random periodic breaks.
} 
market gains. Hence, $\mathrm{CGH}$ test whether momentum profits depend on market states. They find strong evidence that CRSP era momentum profits depend on the state of the market. We verify these findings in the Victorian era - momentum profits are higher following high long run (3-year) market returns and lower following low long run market returns - providing support of CGH.

Momentum cycles may reflect changing risk over the business cycle. Chordia and Sivakumar (2002) [CS] show that macroeconomic instruments commonly used for measuring macroeconomic conditions can explain a large portion of momentum profits. CS argue that intertemporal variations in the macroeconomic risk factors are the main sources of momentum profits. After examining the data in a different way, CGH disagree the findings of CS. We find Victorian-era momentum profits are correlated with market state but unrelated to economic expansion and contraction cycles - and so the high returns to momentum strategies are difficult to reconcile as being compensation for macroeconomic risk.

The historical fact that momentum cycles depend on past market returns and are likely to continue when the cost of investment capital is high suggests that these are times when "smart money," i.e., the capital available to arbitrageurs, is in limited supply relative to available investment opportunities created by “dumb money," i.e., capital available to investors whose behavior is subject to behavioral biases documented in the literature. It would appear that the limits of arbitrage discussed in Shleifer and Vishny (1997) may be particularly binding following up markets, an empirical regularity in the data spanning over 140 years.

\section{B. Rest of the paper}

Our main results - that momentum cycles are consistent across centuries and can be explained by a proxy for the availability of arbitrage capital - are presented in Section 4. Before we present our main findings, we first selectively review momentum literature in Section 2 and document the similarities between the Victorian and CRSP era momentum in Section 3. After establishing the similarities between eras, in Section 4 we formally test whether the momentum profit cycles during the Victorian era and those of the CRSP era share the same properties. Section 5 reports some measures of trading costs for the London market and Section 6 concludes the paper. Technical Appendices provide details about the 
unique data set and the new statistical procedures we apply to momentum duration cycles.

\section{Related Literature}

In what follows we provide a selective survey of the literature, emphasizing only the findings relevant for the analysis in the current paper. ${ }^{5}$

Although several early empirical studies of the efficient market hypothesis examined relative strength strategies, there was little consensus regarding the profitability of such strategies. Levy (1967) claimed that buying stocks when their prices are substantially higher than their 27 weeks moving average resulted in superior profits. Jensen and Bennington (1970) challenged this claim by showing that Levy's trading rules did no better than buy and hold strategies suggesting that Levy's findings could be subject to a data mining bias. In contrast, Fisher Black (1973) found that Value Line rankings that relied, among other things, on relative strength (of the stocks in the industry relative to the composite stock index) had value. Grinblatt and Titman (1989) found that mutual fund managers exhibited a tendency to buy past winners their buy decisions appeared to rely on the existence of price momentum. Lehmann (1990) used a clever portfolio strategy to exploit short run reversal and showed that it is an economically interesting phenomenon. Lo and MacKinlay (1990) examined the sources of momentum profits by analyzing Lehmann's (1990) portfolio strategy. ${ }^{6}$

Jegadeesh and Titman (1993) designed a clever trading strategy that we follow in this paper. Jegadeesh and Titman's (1993) strategy relied on relative price momentum that was well defined and has been replicated by other researchers. ${ }^{7}$ A vast number of studies have confirmed the Jegadeesh and Titman (1993) finding using data from markets in a number of countries - United States, Europe, and emerging economies (see e.g. Rouwenhorst (1998)). ${ }^{8}$ Using a sample from 1973 until February 2008, Asness et al. (2008) study (value and) momentum in five major asset classes: (i) stock selection within four major countries, (ii) country equity index selection, (iii) government bond selection, (iv) currency selection, and

\footnotetext{
${ }^{5}$ For a recent comprehensive survey of the literature, see e.g. Jegadeesh and Titman (2005).

${ }^{6}$ See Jegadeesh and Titman (2005) for a comprehensive survey of the momentum literature.

${ }^{7}$ Jegadeesh (1990) showed short term reversal and medium term continuation in returns in the cross section using regression methods.

${ }^{8}$ Chui, Titman, and Wei (2007) note that Korea, Japan, and Taiwan are exceptions.
} 
(v) commodities. They provide ubiquitous evidence on the excess return to value and momentum, extending the existing evidence to government bonds, currencies and commodities. The consensus appears to be that there is reversal in the short run - i.e., past Winners lose relative to past Losers during the first month following portfolio formation; continuation during the intermediate term - i.e., past Winners continue to win relative to past Losers during the 2 to 12 months following portfolio formation; and long run reversal - i.e., past Winners lose relative to past Losers over 36 to 60 months following portfolio formation. The evidence is stronger for short term reversal and intermediate term continuation. Korajczyk and Sadka (2004) show that momentum profits cannot be explained away by transactions costs. Given their estimates of visible and invisible transactions costs, find that the abnormal returns to some of the momentum strategies disappear only after about $\$ 5$ billion of money chases them.

A variety of explanations are offered for these relations. They range from data issues, such as microstructure and data snooping biases (Boudoukh et al. (1994), Conrad and Kaul (1989), Lo and MacKinlay (1988), to rational risk-based explanations (Conrad and Kaul (1998) Berk et al. (1999), Chordia and Shivakumar (2002), Bansal et al. (2002), to behavioral explanations of irrational behavior on the part of investors (DeBondt and Thaler $(1985,1987)$, Jegadeesh and Titman (1993), Daniel et al. (1998), Barberis et al. (1998), Hong and Stein (1999), Hong et al. (2000), Lee and Swaminathan (2000), Grinblatt and Han (2002), among others). Several theories have been advanced to explain this phenomenon. They can be put into two classes: those that rely on investor psychology affecting stock prices and others that rely on the changing nature of real investment options available to firms. Daniel, Hirshleifer, and Subrahmanyam (1997) (henceforth DHS), Barberis, Shleifer and Vishny (1998) (henceforth BSV), Hong and Stein (1999) (hereafter HS) and Grinblatt and Han (2005) (henceforth GH) are some of the notable papers falling in the former class. DHS assume overconfidence and self attribution leads to momentum from overreaction that subsequently corrects resulting in reversal. BSV assume conservatism and extrapolation on the part of investors and show that will lead to continuation in the intermediate term and reversal in the long run. Hong and Stein assume slow information diffusion and positive feedback trading and show it can lead to momentum and subsequent reversal. GH argue that the tendency of some investors to hold on to their losing stocks, driven by “prospect theory" of Kahneman and Tversky (1979) and "mental accounting" of Thaler (1980), 
can lead to slower diffusion of information and price momentum in stocks. The commonality among all these models is they all rely on biases in how investors process information.

Berk, Green and Naik (1999) (BGN), Carlson, Fisher and Giammarino (2004), Johnson (2002), Chen and Zhang (2007), and Sagi and Seasholes (2007) fall in the latter class. BGN pioneered the latter line of thinking by showing that when firms make optimal investment choices, their assets and investment options change in a predictable way affecting their life cycle risk characteristics and that can also cause price momentum in stocks.

\section{Momentum in the Victorian and CRSP Eras}

This study makes use of a new data set consisting of the closing prices, dividends and shares outstanding of 1,808 stocks (equity) listed in London between 1866 and 1907, compiled by the authors from late $19^{\text {th }}$ and early $20^{\text {th }}$ Century financial publications. As can be seen from Figure 2, the number of stocks we use to form momentum portfolios varies from 126 in 1866 to 1074 in 1907. The number of stocks declines from 985 in 1903 to 544 in 1904, due to a number of industries vanishing from the quotation list, only to reappear in 1905. In Appendix A, we provide a detailed description of the unique and new hand-collected historical data.

In this section, we document the similarities between momentum profits in the CRSP and Victorian Eras. One of the salient features of momentum in modern markets is that the return to momentum strategies varies with changes in formation and holding periods. We begin by verifying the similarity of risk and return at the intermediate term before comparing the term structure of CRSP and Victorian momentum profits.

\section{A. Intermediate Term Momentum in Stock Prices}

The structure of this section follows that of Jegadeesh and Titman (1993), henceforth referred to as JT. It is worth recalling the trading strategies considered by JT. The main motivation is the premise that if stock prices either overreact or underreact to information, then profitable trading strategies that select stocks based on their past returns will exist. Therefore strategies examined 
by JT, consider selecting stocks based on their returns over the past 1 through 4 quarters. To increase the power of the tests, the strategies include portfolios with overlapping holding periods. Therefore, in any given month $t$, the strategies hold a series of portfolios that are selected in the current month as well as in the previous $\mathrm{K}-1$ months, where $\mathrm{K}$ is the holding period. ${ }^{9}$ Specifically, a strategy that selects stocks on the basis of returns over the past $\mathrm{J}$ months and holds them for $\mathrm{K}$ months is referred to as a J-month/K-month strategy. It is constructed as follows: At the beginning of each month the securities are ranked in ascending order on the basis of their returns in the past $\mathrm{J}$ months. Based on these rankings, JT grouped stocks into deciles and formed a value-weighted portfolio of stocks within each decile. Since the number of stocks available to us during the Victorian age is smaller, we will work with a coarser grid, namely top, middle and bottom thirds - or small, medium and large. The top portfolio is called the "losers" and the bottom is called the "winners". In each month $t$, the strategy buys the winner portfolio and sells the loser portfolio, holding this position for K months. ${ }^{10}$

Let us recall that JT used constructed momentum portfolio returns over the 1965 to 1989 period using data from the CRSP daily returns. All stocks with available returns data in the J months preceding the portfolio formation date are included in the sample from which the buy and sell portfolios are constructed. Table 1, similar to Table I in JT, reports the average returns of the different buy and sell portfolios as well as the zero-cost, winners minus losers, portfolios constructed using our data set. JT have a larger set of 32 strategies, while our selection strategy is constrained by the smaller set of stocks and less frequent trading. JT find that the returns of all the zero-cost portfolios are positive and statistically significant except for the 3-month/3-month strategy. Our historical results reported in Table 1 are the same. The weakest case is the $3 / 3$ strategy, which is not statistically significant.

The most successful zero-cost strategy in JT is the $J=12 / K=3$ months strategy which yields $1.31 \%$ per month. Our most profitable strategies are also of the same type $(\mathrm{J}=13 / \mathrm{K}=3$ and $\mathrm{J}=9 / \mathrm{K}=6$ being quite similar) although they yield only $.5 \%$ per month. Moreover, the 9-month formation period produces returns of about $.5 \%$ per month regardless of the holding period. This

\footnotetext{
${ }^{9}$ As noted at the beginning of section 2, we considered 28-day periods as months in our calculations. Moreover, when considering a year, i.e. $\mathrm{K}=12$ months in JT, we used 1328 -day periods.

${ }^{10}$ As mentioned earlier, if a stock, included in a momentum portfolio, vanishes from the database when the portfolio is formed, but does not reappear at any future point in time, we remove it. When a stock does reappear at a later time, we interpolate its price in computing the return on the momentum portfolio.
} 
is to be expected since we use a coarser partition of stocks based on past returns (tercile instead of decile as in JT). While the order of magnitude of monthly returns is less than half that reported in the JT paper, the statistical significance is about the same.

To assess whether momentum strategy profits are abnormally high, it is necessary to examine their exposure to systematic risk. We follow the JT approach in focusing all our remaining analysis on the 6-month/6-month strategy, and thus in the rest of this paper the length of a period is 6 months. This has the advantage that we are dealing with equally spaced return observations, which makes the analysis of factor models and temporal dependence easier.

Table 2 reports estimates of the two most common indicators of systematic risk, the postranking betas of the 6-month/6-month relative strength portfolios and the average capitalizations of the stocks in these portfolios. The table is similar to Table 2 in JT, except for the fact that we have less entries due to the smaller set of strategies considered. We find, (similar to JT) that the beta of the zero-cost winners minus losers portfolio is negative (we find a beta of -0.02 , whereas JT have a beta of -0.08), since the beta of the portfolio of past losers is higher than that of the portfolio of past winners. The average capitalizations of the stocks in the different portfolios show that the highest and the lowest past returns portfolios consist of smaller than average stocks, with the stocks in the losers portfolios being smaller than the stocks in the winners portfolio. This evidence is consistent with JT, suggesting positive risk adjusted returns on average for the momentum portfolio.

Next, we examine the profitability of the 6-month/6-month strategy within subsamples stratified on the basis of firm size. Size is sorted in lower, middle and highest thirds. Panel A and B of Table 3 report average returns and Jensen's CAPM alphas, and Panel C reports CAPM regressions augmented with a NBER Business Cycle dummy. ${ }^{11}$

We note in Panel B of Table 3 that all the CAPM alphas are negative. A robustness check reported in Table 4 shows that this is due to our treatment of missing data. Recall that we set the last monthly return to $-99.99 \%$ whenever a firm vanishes from our sample

\footnotetext{
${ }^{11}$ Consensus U.K. business cycle dates are not available for this time period. Therefore, we use NBER business cycle dates as proxies for the trans-Atlantic business cycle. However, there is considerable evidence that the U.S. and U.K business cycle was correlated during the Victorian era. U.S. and U.K. industrial production and per capita GDP had correlations of .22 and .25 respectively. Historical consumption data is unavailable but average U.K. household earnings grew at a rate of $1.55 \%$ in years without NBER contractions and $1 \%$ in years with an NBER contraction. See Officer $(2008 \mathrm{a}, \mathrm{b})$.
} 
after portfolio formation. This grossly understates the actual returns. In Table 4, we compute returns under the alternative assumption that firms leave the database at random and do not set the last monthly return to $-99.99 \%$. This alternative method ignores the survivorship issue, as is done in JT. Under the alternative treatment of missing data, half the portfolios have positive alphas and half have negative alphas - as is found with modern era data. Thus, we can be confident that the negative CAPM alphas are due to our treatment of missing data.

As JT note, measuring relative strength profits on size-based subsamples allows us to examine whether the profitability of the strategy is confined to any particular subsample of stocks. This analysis also provides additional evidence about the source of the observed relative strength profits. Table 3 presents the average returns of the 6-month/6-month strategy for each of the subsamples. The results we report are in line with JT. They indicate that the observed abnormal returns are of approximately the same magnitude when the strategies are implemented on the various subsamples of stocks as when they are implemented on the entire sample. Unlike JT, we do not find a particularly strong relationship with firm size. For the zero-cost, winners minus losers portfolio, JT find that the subsample with the largest firms generates lower abnormal returns than the other two subsamples. In Table 3, we find a large return for the medium-sized category, although the large firms in our sample still create the lowest return for the zero-cost, winners minus losers portfolio. When we control for the business cycle, we obtain similar findings, as discussed later. These findings indicate that the relative strength profits are not primarily due to the cross-sectional differences in the systematic risk of the stocks in the sample.

Likewise, Table 5 reports the average returns of the zero-cost portfolio in January versus the rest of the year. JT note that following Roll (1983), there are reasons to expect that the relative strength strategies will not be successful in the month of January. They find that relative strength strategy loses about $7 \%$ on average in each January but achieves positive abnormal returns in each of the other months. We do not find such seasonal patterns, namely the month of January does not yield negative returns in our sample. Moreover, according to statistical tests reported in Table 5, there is, with a few minor exceptions, basically no difference between January and the other months of the year. Our findings essentially confirm the Roll (1983) story. With no capital gains taxes in our time period, we do not expect a January 
effect, as predicted by Roll (1983).

To conclude, we examine again whether our choice of missing data treatment alter the results of the paper. In Table 6, we report the results we obtain for size-sorted average monthly returns and t-statistics for buy minus sell portfolios under our treatment of missing data and the results we would obtain if we relaxed our assumptions by either ignoring the extinction (don't set the last return to $-99.99 \%$ ) or ignoring all missing data by assuming it is missing completely at random (MCAR) and computing results from observable data only. Setting extinct stocks to 99.99\% obviously lowers gross returns but the relative comparisons of portfolios sorted by size or momentum are robust to the treatment of missing data. The t-stats are significant under all treatments of missing data. The results in Table 6 show that our decision to replace missing with $-99.99 \%$ is in fact the most conservative. Moreover, the probability that a firm vanishes appears to be largely independent of past returns. While the actual magnitude of winner and loser alphas depends on the treatment of missing data, the alpha generated from a long winner short loser portfolio is robust to assumptions about missing firm returns.

\section{B. Term structure of momentum returns}

Momentum profits can occur for a variety of reasons even from the perspective of someone who subscribes to the behavioral point of view. For example, if momentum profits are entirely due to slow diffusion of information, then the positive abnormal returns should decay over time to zero. On the other hand, if momentum profits are due to delayed overreaction, momentum profits will likely reverse in sign before decaying down to zero over time. We therefore examine the term structure of the profits to the various momentum portfolios in this section.

We report in Table 7 the average monthly excess return on the momentum portfolios during each of the five years following portfolio formation, after skipping a month. ${ }^{12}$ The loser portfolios' returns almost double from year 1 to year 5. In contrast, the winner portfolios' returns come down somewhat by year 5. Therefore the difference, i.e., the winner minus loser momentum portfolio returns becomes negative in years 4 and 5, and marginally significant. As

\footnotetext{
${ }^{12}$ It is important to note that the results in Table 7 are computed from all available time periods. This means the sample periods vary by column. For example the portfolio return in year 2 after formation is computed by looking at the buy-sell portfolio 14-26 months after formation, so our first "year 2" observation is July 1867June 1868. Our first "year 5" observation doesn't begin until July 1871, however. Therefore the Year 2 column has more observations than the Year 5 column. This is also why the various columns do not add up.
} 
can be seen from Figure 4, a dollar invested in the 6 month Winner portfolio can be expected to grow steadily over time to 1.15 dollars in 5 years; and a dollar invested in the 6 month Loser portfolio can be expected to decline to 0.99 dollars after 19 months before rising to 1.08 dollars at the end of 5 years. The difference between the value of a dollar invested in each of the Winner and the Loser portfolios peaked at 7.7 cents on average by the end of the 3 rd year before narrowing down to 6.1 cents at the end of 5 years (see Figure 5). So, while the evidence of long run reversal exists, it is not as dramatic as that reported in Jegadeesh and Titman (2001). We can reject the hypothesis that momentum returns in years 2 to 5 are the same as that during year 1, in favor of the alternative that they are smaller at conventional levels of significance.

Since our historical data is sampled every 28 -days, therefore one year is 13 "months". We wish to test the null hypothesis that the winner minus looser (WML) portfolio exhibits no momentum or reversal. We begin by forming a $T \times N$ matrix $X$ where the $(t-t h ; n-t h)$ element is equal to the month $t$ annual return on the WML portfolio formed $n$ years earlier. Let $X(t, n)$ be the holding period return from month $(t-12)$ to month $t$ on the WML portfolio formed $n \times 13$ "months" earlier. Under the null hypothesis that WML returns are independent of time since formation, each column of $X$ should have the same expectation. We test this hypothesis by subtracting the year $1 \mathrm{WML}$ return from the year 2,3,4 and 5 returns to form a new $\dot{X}$ matrix such that $\dot{X}(t, n) \equiv(X(t, n+1)-X(t, 1))$ : Therefore $\dot{X}(t, n)$ measures the difference between the holding period return from month $(t-12)$ to month $t$ on the WML portfolio formed $(n+1)$ years earlier and the WML portfolio formed $(n+1)$ year earlier. Under the null hypothesis of no momentum or reversal $\dot{\mathrm{X}}(t, n)$ should have expectation zero. Table 8 reports the mean return of the WML portfolio 1 through 5 years after formation. We test of the null that $E[\dot{\mathrm{X}}(t, n)]=0 \forall t$ and $n$ via a Wald test. ${ }^{13}$ The results in Table 8 show strong rejections of the null that average monthly returns are equal in years 1 through 5. It is interesting to note the pattern of mean returns: 0.0034 after one year, 0.0016, 0.0006 after two and three years and finally -0.0006 and -0.0015 after years four and five. This reaffirms the finding of short run reversal, medium term continuation, and long run reversal in past winners minus past losers portfolio returns.

\footnotetext{
${ }^{13}$ More specifically, consider: $g=\left(\sum_{t}[\dot{\mathrm{X}}(t, 1)] / T, \ldots, \sum_{t}[\dot{\mathrm{X}}(t, 4)] / T\right)$; then the test statistic is $W=$ $g \operatorname{Cov}(g)^{-1} g$, where under the null $W \sim \chi^{2}(4)$, asymptotically. Since the elements of $\dot{X}$ are serially correlated, we use a HAC estimator for $\operatorname{Cov}(g)$ using Newey-West with 13 lags.
} 
The lower panel of the Table shows some pairwise test results and we note that year 1 is significantly different from all other years. The average monthly returns after 2 and 3 years appear to have the same mean, and so do years 3 and 4 as well as 4 and 5 .

It should be noted that the above test may be biased by missing data. In particular, when an asset vanishes from our data set we set its return to $-99.9 \%$ at the time of extinction and replace missing data with interpolated data when possible. However, we verified that the momentum in years 1 and 2 and reversal in years 4 and 5 are robust to missing data treatment, and we find indeed they are. ${ }^{14}$

\section{The State of the Market, the Economy and Momentum}

Recall that Cooper, Gutierrez and Hameed (2004) appeal to the theory of DHS to predict differences in momentum profits across states of the market, like bull and bear markets, as aggregate overconfidence should be greater following market gains (DHS and Gervais and Odean (2001)). With overconfidence higher following market increases, overreactions will be stronger following up markets, generating greater momentum in the short run. The HS model is also based on initial underreaction to information and subsequent overreaction, which eventually leads to stock price reversal in the long run.

For each month, between 1929 and 1995, CGH denote the state of the market as Up (Down) if the markets trailing three-year return is positive (negative) on that date. They compare the returns of momentum portfolios formed during $U p$ and Down markets and conclude that momentum was largely an up market phenomenon during the CRSP era. The London market index was seldom down in the 3-year windows during our historical sample period. We therefore take a slightly different definition of up and down markets. By CGH's definition, 85 \% of their observed market states were UP markets. We denote Victorian market states as follows $U p=$ market index return over the past 3 years in the top $85 \%$ of sample returns, and Down = market

\footnotetext{
${ }^{14} \mathrm{We}$ did this as follows. If survival rates differ across buy and sell portfolios this may bias our reversal tests. We evaluate the effect of missing data on the results in two ways. First we simply compute the probability of extinction. If the probability of extinction is independent of past return the momentum results should be robust to our choice of missing data treatment. We find survival probabilities that a stock remains in the data set, nmonths after formation, for buy and sell portfolios. The difference between survival rates in buy and sell portfolios is very small. We also computed the event time returns of WML portfolios under our treatment of missing data and an alternative MCAR treatment that simply ignores securities that vanish. If survival depends on past return the MCAR estimates should differ. Instead the MCAR results are statistically indistinguishable from our treatment of missing data.
} 
index return over the past 3 years in the bottom $15 \%$ of sample returns. In Table 9 we report the evidence of momentum and market states in our sample and compare our results to CRSP era results computed with our sorting method. Recall that $\mathrm{CGH}$ sort stocks into deciles while we sort into terciles. The choice of tercile sorts instead of deciles, weakens the relationship between market state and momentum in the CRSP era but the difference between UP and DOWN market states, remains.

Average profits and risk adjusted returns by market state are computed by taking the profits of each zero-cost momentum portfolio (winner minus loser terciles) for each formation date and averaging across all formation dates that qualify for a particular market state. The average monthly profit of the momentum portfolio formed at time $t$ is:

$$
\operatorname{AvgRET}_{t}=\left[\sum_{k=t+1}^{t+6} r_{k}\right] / 6
$$

where $r_{k}$ is the return of the 6-6 winner minus loser momentum portfolio formed at time $t$. We follow CGH and also report the average CAPM alpha across market states. The CAPM alpha of the momentum portfolio formed at time $t$ is:

$$
\alpha_{t}=\left[\sum_{k=t+1}^{t+6} r_{k}-\widehat{\beta}_{t}\left(r_{m, k}-r_{f, k}\right)\right] / 6
$$

where $\widehat{\beta}_{t}$ is the OLS estimator of a CAPM regression, using data from $t+1$ to $t+6$.

Table 9 reports the mean monthly profits and mean CAPM alphas by market state. The Up market average monthly profit is computed by taking the average of all $A v g R E T_{t}$ for formation time $t$ that corresponds to an Up market state. Down market results are computed in the same manner. Since the average returns are computed from overlapping holding periods, all t-stats are computed with the Newey and West (1987) HAC procedure using 5 lags.

$\mathrm{CGH}$ find that momentum profits depend on the state of the market, as predicted. From 1929 to 1995 , the mean monthly momentum profit following positive market returns is $0.93 \%$ in their sample, whereas the mean profit following negative market returns is $-0.37 \%$. The upmarket momentum reverses in the long run.

In Table 9 for the 6/6 winner minus loser portfolio, we find that the average monthly return following an $U p$ market is 40 bp per month in the CRSP sample, and 38 bp per month in our sample. The corresponding numbers following a Down market are -28 bp per month and 15 bp per month respectively. Momentum profits are higher following $U p$ markets than Down 
markets in our sample just like in the CRSP sample but while the difference is statistically significant in the CRSP sample, it is not significant in our sample. We also find similar patterns for Jensen's alpha.

Next, we examine whether commonly used macroeconomic instruments for measuring market conditions can explain a large portion of momentum profits, following the methods used in CGH. Following Chordia and Sivakumar (2002), we construct a factor model for expected returns:

$$
r_{t}=a+b_{1} M k t_{t}+b_{2} \text { Divyld }_{t}+b_{3} \text { Term }_{t}+b_{4} \text { Default }_{t}
$$

where the return on the 6-6 momentum portfolio (i.e., Winner minus Loser monthly rate of return) is projected onto Divyld ${ }_{t}$ : lagged dividend yield on the market index, Term $_{t}$ : lagged term spread [yield on British consol - yield on 30-day bankbill], and Default $_{t}$ : lagged default spread [yield on risky bonds (portfolio of British RR bonds) - yield on British consol]. ${ }^{15}$

We use two methods to evaluate the ability of the factors to explain momentum profits. In Panel A of Table 10, we report the slope coefficients, the test statistic for the hypothesis that $b_{2}=b_{3}=b_{4}=0$ and the proportion of variation collectively explained by the Divyld; Term and Default factors. The latter is obtained via the $R^{2}$ of the CAPM residuals regressed on Divyld; Term and Default: For most 6-6 momentum portfolios, we can soundly reject the null that the betas on the macroeconomic factors are collectively equal to zero. It should be noted that CS use a 3 factor model with no market index. We add the market index for the regression that reports factor loadings and proportion of extra variation explained by CS's 3 factors (panel A of Table 10). In Panel B of Table 10, we sort on CS's three factors model (no market index). However, the proportion of variation explained by the macro factors is extremely small. Collectively, the macro factors explain less than $3 \%$ of the time series variation in portfolio returns and in the case of the largest stocks less than $0.3 \%$.

CGH employ a clever double sort methodology to illustrate the relative influence of momentum and factor premium on portfolio returns. For each stock and time period, they compute the factor loadings via an OLS regression over the trailing 60 months. With time $t$ beta estimates in hand, they compute the expected return of each stock over the future 6 months given current betas and actual future realizations of the factors. They then sort stocks into portfolios

\footnotetext{
${ }^{15}$ We computed the yields on the British consol and British railroad bonds from quotations in the Course of the Exchange. The yield on 30-day bank bills was collected from The Economist.
} 
based on momentum and factor model expected return. We form historical portfolios via this method and report the results in Panel B of Table 10. Winner minus Loser portfolios within each of the three predicted return categories earn positive returns on average; the average returns are statistically (t-statistic of 3.75) and economically (54 bp per month) significant within the high predicted returns group. Further, within the Loser and Middle groups, stocks with high predicted returns earn lower returns than stocks with low expected returns, and they are jointly significantly different from zero. Hence, the findings with Victorian era data support the results of $\mathrm{CGH}$, although our evidence appears somewhat weaker.

\section{Business Cycles and Momentum Profits}

During the Victorian age there were at least as many business cycles as in the current CRSP data; and the depressions of those days have been characterized as more severe. ${ }^{16}$ We therefore examine how momentum profits are related to business conditions (expansions and contractions; as well as past stock market returns). To do so we turn our attention now to Tables 11 and 12. In Table 11, we report the results of the regression:

$$
r=a+b_{1} M k t+b_{2} G D P
$$

where $r$ is the return on buy portfolio minus the return on sell portfolio and GDP is the demeaned real per capita GDP growth. We examine this regression for small, medium and large stocks. In none of the cases do we observe any significantly positive exposure of the momentum profits to GDP growth rate risk; in fact the point estimates of the slope coefficient for GDP growth rate is negative for all size classes.

To complement this, we also report in Table 12 a correlation matrix for the following variables: real per capita GDP growth, buy-sell portfolio annual returns for small, medium and large stocks, the average cross-sectional monthly stock return standard deviation, market excess returns and UK short and long-term rates obtained from Ordinary Funds. The correlations are computed using annual data. The first column of Table 12 shows that the annual returns of the buy-sell portfolio for small stocks features very negative correlations with GDP growth (around 30\%), as well as the excess market returns and the long term rate. The medium-sized firms feature similar negative correlations except for the long rate which

\footnotetext{
${ }^{16}$ There were 10 NBER recessions between 1866 and 1907 and 10 between 1946 and the present.
} 
is positively correlated with buy-sell portfolio annual returns for medium firms (more than $35 \%$ ). Finally, the Buy-sell portfolio annual returns for large firms also show negative correlation with GDP growth, although the correlation monotonically declines with the size of the firms.

\section{Momentum Cycles and Limits to Arbitrage}

So far we have established that the momentum strategy has generated abnormal returns for over 140 years. When a strategy as simple as buying past winners and selling past losers produces an almost free lunch, i.e., high returns on average with little or no systematic risk, we are left with several possibilities.

First, for the Victorian and much of the CRSP era, investors may not have been aware of the existence of the almost free lunch. However, given the attention in the academic and trade literature it has received, investors will likely arbitrage away any future momentum profits. This does not appear to be the case. The average return on our WML portfolio since 1990 is actually higher than the return before 1990 .

Second, the almost free lunch may not exist. That is, the apparent high returns may be a spurious artifact of data mining that is unlikely to show up in future data. While a number of studies document the existence of price momentum in stocks in the United States and other countries, almost all of these studies use data from the same post World War II period and hence their findings cannot be viewed as being entirely independent of each other. Again, this is unlikely to be the case. Unlike cross-country comparisons, returns in Victorian England are independent of modern returns. This paper has increased the time series of available momentum returns by $50 \%$ without altering the abnormal returns.

Third, the lunch may not be free. Momentum strategies may generate high returns on average but expose investors to low returns during severe economic contractions. Such business cycle risks may not be discernable by looking at the Post-War CRSP era data alone. If momentum portfolios expose investors to occasional severe real risks at business cycle frequencies we may need to observe returns in more than the handful of relatively benign PostWar cycles before these risks become apparent. Historical Victorian age data helps us evaluate 
this hypothesis by observing momentum returns across several business cycles of varying levels of severity. Recessions were both more frequent and more severe during our Victorian era sample period. The NBER business cycle chronology includes 10 troughs during the 42 years between 1866 and 1907 compared to 10 troughs in the 63 years since World War II. ${ }^{17}$ The cycles were more severe as well. The standard deviation of annual per capita UK GDP growth was $4.5 \%$ between 1866 and 1907 and 3\% between 1946 and 2007 (see Johnston and Williamson (2008)); yet the momentum profits persist.

What then explains the persistence of momentum? We think the answer lies in the cycles apparent in Figure 1. While momentum portfolios do generate high returns orthogonal to market and business cycle risks, the momentum strategy also exposes investors to high variance and frequent losses. Momentum trading involves active trading by relatively sophisticated investors. If many well capitalized sophisticated investors followed momentum strategies at the same time we would expect their collective action to squeeze the profit out of the strategy. The frequent loses associated with our momentum strategy makes it difficult for investors who use other people's money or leverage to drive momentum profits out of the market.

The WML momentum portfolio is self-financing but traders would have to post margin against losses. The lag-6, hold-6 WML portfolio suffered drawdowns of $10 \%$ or more once every 3.4 years during the CRSP era. These drawdowns were quick - lasting only 4.5 months on average - and sharp - the WML portfolio lost an average of $17.5 \%$ per drawdown. Most professional money managers who rely on investors for financing cannot lose one third of their capital in a few months time without being driven out of business by redemption requests. Given the frequent drawdowns in the WML portfolio any money manager employing even 2:1 leverage would be unlikely to last more than a handful of years. This is the well known "limits to arbitrage" explanation of persistent anomalies. Momentum strategies look like winners for unlevered investors, but without leverage the number of sophisticated investors must be large relative to noise traders before the anomaly vanishes. In models such as Shleifer and Vishny (1997) rational but capital constrained investors can generate cycles if losses in the WML portfolio force momentum investors to unwind positions to meet liquidation requests. In such a setting momentum profits depend on the relative supply of capital to noise and

\footnotetext{
${ }^{17}$ The NBER business cycle chronology is for the U.S. No NBER equivalent exists for the U.K. but the U.K. and U.S. business activities were highly correlated during the Victorian Age.
} 
arbitrage traders. Momentum profits will be high when times are good and plentiful noise traders provide profit opportunities or when arbitrage capital is scarce and few sophisticated traders compete.

If volatility limits arbitrage, we should observe cycles in momentum and be able to predict when momentum profits will vanish. In this section, we examine the cyclicality of the WML returns and formally test whether momentum profit cycles from the Victorian and CRSP eras share the same features and are predictable by the same variables.

We test these hypotheses in two ways. First, we establish the fact that momentum portfolios have been subject to similar cycles since the 1860s. We examine return distributions and the duration spells of momentum profit and losses from both eras. We test the underlying null hypothesis that the momentum portfolio returns and duration spells are drawn from the same distributions across eras. Second, we model the duration dynamics as a function of the state of the economy, the market return and a measure of scarcity of capital. If capital constraints limit arbitrage, we would expect a measure of capital scarcity to predict the duration of cycles.

We start with the momentum return distribution during both the Victorian and CRSP eras and test whether the momentum return is white noise for both eras. Before we estimate AR coefficients, we need to remind the difference in data structure between eras - four weeks for the Victorian era and one month for the CRSP era. Thus, $\rho_{13}$ of the Victorian era should be compared with $\rho_{12}$ of the CRSP era. So, we restrict our attention on $\rho_{\mathrm{k}}$ to $\mathrm{k}=1,2, \ldots, 6$ for monthly data and to $\mathrm{k}=1,2, \ldots, 4$ for quarterly data. Table 13 shows the estimated autocorrelations and t-stats. The Ljung-Box test in Table 14 shows the p-value for the null that $\rho_{\mathrm{k}}=0$ for all $\mathrm{k}$. The Box-Pierce test in Table 14 shows the $\mathrm{p}$-value for the null that $\rho_{\mathrm{k}}^{\text {Victorian }}=$ $\rho_{\mathrm{k}}^{\text {CRSP }}$ for all $\mathrm{k}$. For monthly data, we can reject that $\rho_{\mathrm{k}}=0$ for all $\mathrm{k}$ for the CRSP era with $95 \%$ confidence level. And, for quarterly data, we can reject that $\rho_{\mathrm{k}}=0$ for all $\mathrm{k}$ for both eras with 95\% confidence level. Furthermore, for both monthly and quarterly data, we cannot reject the null that $\rho_{\mathrm{k}}^{\text {Victorian }}=\rho_{\mathrm{k}}^{\text {CRSP }}$ for all $\mathrm{k}$. Hence, the momentum returns are different from white noise and both eras share the same AR features.

Next, we look at duration spells. We construct the sequence of duration spells for each era. For example, if the monthly series of momentum profit is $(0.01,-0.04,0.01,0.01,0.01,-0.02$, $0.02)$, the sequence of duration up is $(1,3)$ and the sequence of duration down is $(1,2)$. We apply this method for both eras and get 133 observations for the Victorian era and 224 observations of 
CRSP era. Table 15 summarizes the empirical distribution of duration spells for both periods. We can see that empirical distributions are roughly similar.

Suppose we call the sequence of duration up (down) duration spell $\mathrm{D}_{\mathrm{iU}}\left(\mathrm{D}_{\mathrm{iD}}\right)$ and we want to test the null hypothesis that: $\mathrm{H}_{0}: \mathrm{D}_{\mathrm{iX}} \sim \mathrm{F}$, for all $\mathrm{i}$ during the Victorian and CRSP eras for $\mathrm{X}=\mathrm{U}$ or $\mathrm{D}$, where $\mathrm{F}$ is some duration distribution. We essentially adopt two strategies in testing this null hypothesis. The first is a non-parametric approach, where we do not explicitly specify a duration distribution. The second is parametric, and relies on a specific parametric distribution. Both strategies have advantages and disadvantages. The non-parametric approach has the obvious advantage that we do not have to select a distribution for the duration spells, whereas the parametric approach not only tests the null hypothesis of equality across the two eras, but also whether we actually selected a distribution that properly describes the duration spells. Hence, the parametric approach tests a joint hypothesis, the null of identical duration distributions and the null that the chosen duration distribution $\mathrm{F}$ is correctly specified. In contrast, the non-parametric approach - while distribution-free - requires the duration spells to be i.i.d. Since, we examine the sequence of up/down durations, this may not be an unreasonable assumption. Moreover, the non-parametric tests typically are less powerful, as are many nonparametric tests, each having particular power properties again violations of the null hypothesis. For the non-parametric tests we consider four tests, they are (a) the Mann-Whitney-Wilcoxon test, (b) Kolmogorov-Smirnov test, (c) Cramer-von Mises test, and (d) Kuiper test. The MannWhitney-Wilcoxon test is that the two samples are drawn from a single population, and therefore that their probability distributions are equal - although it is sometimes referred to as a test of equal medians. The Kolmogorov-Smirnov (K-S) and Cramer-von Mises test statistics quantify a distance between the empirical distribution functions of the two era samples. The null distribution of these statistics is again calculated under the null hypothesis that the samples are drawn from the same distribution. Finally, the Kuiper test is a variation of the K-S test that is more sensitive to tail behavior. Details about the tests appear in Appendix B. In Table 16, we report the results of the aforementioned tests. Entries to the table are p-values for the null hypothesis that the duration spells during the Victorian and CRSP eras are drawn from the same population. 
The results are fairly easy to summarize. Overall we find overwhelming evidence in favor of the null hypothesis that both eras feature duration spells that come from the same distribution. The distribution of momentum cycles has remained similar since the $1860 \mathrm{~s}$.

In our parametric tests, we have to select an explicit distribution for the durations. We consider the following distributions: (a) the exponential, (b) Gamma, (c) Weibull. In Figure 6, we plot the empirical duration up distribution against the three aforementioned functional forms of duration distributions. In Figure 7, we plot the empirical duration down spell distribution against the three aforementioned functional forms of duration distributions. Clearly, the exponential distribution inadequately describes the data, whereas the other two are more plausible. When we estimate the latter two, we find parameter estimates that are roughly the same across the two eras. In Table 17, we report the Maximum Likelihood estimates and standard errors. We also tested whether the parameters across the two eras are identical and cannot reject the null that they are equal (details available upon request).

We are confident that the momentum strategy generates similar return and duration distributions in both eras. The question remains: can we explain momentum? We hypothesize that the observed cycles are caused by changes in the relative supply of capital available to sophisticated momentum traders and noise traders. We predict that a measure of the scarcity of capital will affect the likelihood of duration spells. Specifically, we use the real risk-free rate as a proxy for the scarcity of capital. When the risk-free rate is high we expect the capital available to momentum traders to be in short supply and the cost of leverage to be high. With relatively few sophisticated traders competing for momentum profits we expect profit (UP) cycles to last longer and loss cycles (DOWN) to be shorter. Thus, we expect the real risk-free rate to effect the duration of returns in different directions depending on whether we are in an UP or DOWN cycle. This is one possible explanation on momentum cycle through the demand effect.

One the other hand, we can think about naïve investors as liquidity providers. As the funds from naïve investors increase, the sophisticated momentum traders may prolong momentum profits. This is another explanation on momentum cycle through the supply effect. We use past stock returns as a proxy of the size of naïve investors. Note that, in Table 9, we showed that the momentum profits are significantly high after UP markets. There is also a demand effect, the willingness of sophisticated investors to sustain momentum strategies. In line with the previous empirical analyses, we estimate duration distributions with state variables. Specifically, we 
model the parameters in the Weibull and Gamma distributions as $\lambda=\exp (-$ intercept $\beta$ 'state variables) with the risk-free rate, and the stock market state as covariates. The details of the procedure appear in Appendix B. Under both parametric assumptions, the expected duration is inversely related to $\lambda$. Thus, the positive (negative) sign of $\beta$ can be interpreted as the positive (negative) relationship between state variables and durations.

The results are reported in Table 18. As predicted the point estimate on the risk-free rate has a positive effect on the duration of UP cycles and a negative effect on the duration of DOWN cycles. Regardless of specification, the point estimates always have the predicted sign although often insignificant from zero - particularly for impact of past market returns on momentum durations. In Table 19 we formerly test the null hypothesis that the level of the risk-free rate differs across UP and DOWN cycles. We test this hypothesis by pooling the UP and DOWN cycles and allowing the all coefficients to vary with an interaction dummy equal to one if the observation is a DOWN cycle. Regardless of era or specification, the effect of the risk-free rate is always significantly lower in DOWN cycles. Hence, when capital is plentiful, the probability of momentum traders suffering losses is significantly lower than when capital is scarce.

\section{Trading Costs}

In Tables 20 and 21, we turn our attention to transaction costs. It is indeed worth investigating how costly it would be to sustain a momentum strategy. Korajczyk and Sadka (2004) find that several momentum strategies may not implementable on a large scale when transactions costs are taken into account. Lesmond, Schill and Zhou (2004) find that those stocks that generate large momentum returns are precisely the stocks with high trading costs. Both studies use data, such as intraday data, which is not available in our historical period. We therefore use a simpler approach to gauge the importance of trading costs associated with momentum portfolios.

Table 21 provides data on average bid-ask spreads and average prices of stocks in each of the portfolio categories, whereas Table 20 displays the transition probabilities pertaining to portfolio turnover. Regarding the latter, each cell in the matrix contains the proportion of times that a stock in a given portfolio at time $t$ lands in a given portfolio at time $t+1$ : With respect to trading costs we are most interested in the diagonal values which 
tell us the proportion of times a stock remains in the same portfolio during a new formation. The persistence of stock assignment is generally in the 30-35\% range, which is fairly high compared to current momentum strategies (see e.g. Conrad and Kaul (1998)).

Table 21 also reports the value-weighted bid-ask spread as a percentage of the midpoint for all stocks that are assigned to each portfolio at each date. For example, when the Large Buy portfolio's spread is .02, it means that the value-weighted average bid-ask spread of all stocks purchased or sold from the Large Buy portfolio was $2 \%$ of the midpoint of their bid-ask price. To get an idea of transaction costs, it should be noted that the average bid-ask spread of the Large Buy portfolio was .02. We know that only $1 / 6$ of the portfolio is replaced each period so dividing by $6.02 / 6=.0033$. This is the average per period transaction cost if one paid the full bid-ask spread and bought and sold every stock in the new and old portfolios. However, some of the stocks in the new portfolio were also in the portfolio we formed 6 months ago. From the transition matrix in Table 20, we see that on average $32 \%$ of the stocks in the Large Buy portfolio at any time are also in the same portfolio 6 months later. We would therefore only have to turn over $68 \%$ of the stocks. Multiply .0033 by .68 to get the final back of the envelope estimate of bid-ask costs of roughly 23 basis points per period. This turns out to be the lowest cost. For the other portfolios the costs can be as high as 229 basis points (Small Loser). Hence, these rough measures show that trading costs are high, at least as much as they are in recent history, which makes the profits of momentum not realizable except to those who were making a market in those stocks.

\section{Conclusion}

Among financial market anomalies, price momentum in stocks is generally viewed as posing the greatest challenge to rational asset pricing theories. Shleifer and Vishny (1997) proposed that anomalies like momentum could persist due to the limits to arbitrage imposed by slow movements of capital available to arbitrageurs. The limits to arbitrage arguments imply that when arbitrage capital is in short supply profitable momentum cycles should last longer and breaks in momentum cycles should be shorter. We provide empirical support for this hypothesis by using the level of the interest rate as a proxy for the shortage of available arbitrage capital. 
With increasing globalization, arbitrageurs in one country can exploit with ease profitable trading opportunities in other countries. That makes it difficult to measure shortage of arbitrage capital, since capital can easily move across national borders. We therefore focus on the US market, since the US is a much larger economy than other countries in the world and if the level of the interest rate were to proxy for shortage of arbitrage capital, we should expect it to hold better in the US. We therefore use US stock market data for the 1926 - 2008 period to examine our hypothesis.

Since the level of the interest rate is a poor proxy for the shortage of arbitrage capital, we examine the robustness of our conclusions by checking the validity of our hypothesis using data from an entirely different period of history. We use a new hand-collected data set of the London Stock Exchange which consists of the closing prices, dividends and shares outstanding of 1,808 stocks (equity) listed between 1866 and 1907 (Victorian age). By today's standard, the market was very primitive - and therefore presented an interesting case to examine price momentum. We find strong support for our hypothesis in both the 1926-2008 US stock market data as well as the Victorian age data. In addition, the histogram of the lengths of profitable momentum cycles and breaks in momentum cycles in the two periods are about the same.

When we replicate the trading strategy designed by Jegadeesh and Titman (1993) using Victorian age data, we find compelling evidence for short term reversal, intermediate term continuation and long run reversal in momentum profits. Momentum profits are low following three year down markets and high following three year up markets; and they do not appear to be compensation for economy wide systematic risk, captured by the standard CAPM or macroeconomic factor models. They are higher during economic contractions, a pattern that is different from the post World War II period. Our finding of price momentum during the Victorian age eliminates the possibility that price momentum may be an artifact of data mining.

That is the systematic pattern across data spanning several centuries and is consistent with explanations based on overconfidence and an abundance of noise traders relative to availability of arbitrage capital. The defining characteristics of momentum - its cyclicality - is not related to GDP but is predictably linked to the level of the real risk-free rate. The theoretical literature emphasizes the role of "dumb money" and "limits of arbitrage" in explaining price momentum in stocks. Conventional wisdom is that relative abundance of dumb money is typically associated with sustained periods of up markets and low interest rates leading to asset 
bubbles that will be difficult to burst even for arbitrageurs with very deep pockets. Our findings suggest that "dumb money" may be abundant relative to "smart money" even during periods when the risk-free rate is high. 


\section{References}

[1] Annaert, J. and W. Van Hyfte, 2006, Long-horizon mean reversion for the Brussels Stock Exchange: evidence for the 19th century, Discussion Paper, Ghent University.

[2] Asness, C., T. Moskowitz and L. Pedersen, 2008, Value and Momentum Everywhere, Discussion Paper, Chicago and NYU.

[3] Barberis, Nicholas, Andrei Shleifer, and Robert Vishny, 1998, A Model of Investor Sentiment, Journal of Financial Economics 49, 307 - 343.

[4] Berk, Jonathan, Richard Green and V. Naik, 1999, Optimal Investment, Growth Options and Security Returns, Journal of Finance, 54, 1553 - 1608.

[5] Black, Fischer, 1973, Yest, Virgina, There is Hope: Tests of the Value Line Ranking System, Financial Analyst Journal, 29, 10 - 14.

[6] Brav, A., and J. B. Heaton, 2002, Competing Theories of Financial Anomalies, Review of Financial Studies 15, 575 - 606

[7] Carhart, M. M., 1997, On persistence in mutual fund performance, Journal of Finance, $52,57-82$.

[8] Carlson, M., Fisher, A., Giammarino, R., 2004, Corporate investment and asset price dynamics: implications for the cross-section of returns, Journal of Finance 59, 2577 - 2603.

[9] Chan, Louis K. C., Narasimhan Jegadeesh, and Josef Lakonishok, 1996, Momentum strategies, Journal of Finance 51, 1681 - 1713.

[10] Chen, Long and Lu Zhang, 2007, Neoclassical Factors, Working Paper, Ross School of Business, University of Michigan.

[11] Chui, Andy C. W., Sheridan Titman, and K. C. John Wei, 2007, Individualism and Momentum around the World, Working paper, University of Texas.

[12] Chordia, Tarun, and Lakshmanan Shivakumar, 2002, Momentum, business cycle, and time-varying expected returns, Journal of Finance 57, 985 - 1019

[13] Conrad, Jennifer, and Gautam Kaul, 1998, An anatomy of trading strategies, Review of Financial Studies 11, 489 - 519.

[14] Cooper, Michael. J., Roberto C. Gutierrez, and Allaudeen Hameed, 2004, Market states and momentum, Journal of Finance 59, 1345 - 1365.

[15] Daniel, Kent, David Hirshleifer, and Avanidhar Subrahmanyam, 1998, Investor 
psychology and security market under- and overreactions, Journal of Finance 53, 1839 1886.

[16] Daniel, Kent, M. Grinblatt, S. Titman, and R. Wermers, 1997, Measuring mutual fund performance with characteristic-based benchmarks, Journal of Finance 52, 1217 - 1218.

[17] Fama, Eugene F., and Kenneth R. French, 1996, Multifactor explanations of asset pricing anomalies, Journal of Financial Economics 51, 55 - 84.

[18] Frazzini, Andrea, 2006, The disposition effect and under-reaction to news, Journal of Finance

[19] Gabaix, Xavier, 2009, Power Laws in Economics and Finance, forthcoming, Annual Review of Economics.

[20] Gabaix, Xavier, Parameswaran Gopikrishnan, Vasiliki Plerou and H. Eugene Stanley 2006, Institutional Investors and Stock Market Volatility, Quarterly Journal of Economics, 121, 461-504.

[21] Gabaix, Xavier, and Rustam Ibragimov, 2009, Rank-1/2: A Simple Way to Improve the OLS Estimation of Tail Exponents forthcoming, Journal of Business Economics and Statistics.

[22] Gatev, Evan, William Goetzmann and Geert Rouwenhorst, 2006, Pairs Trading: Performance of a Relative-Value Arbitrage Rule, Review of Financial Studies, 19, 797 - 827.

[23] Gervais, Simon, and Terrance Odean, 2001, Learning to be overconfident, Review of Financial Studies 14, 1 - 27.

[24] Goetzmann, William, 1993, Patterns in Three Centuries of Stock Market Prices, Journal of Business, 66, 249 - 270.

[25] Goetzmann, W., R.G. Ibbotson, and L. Peng, 2001, A New Historical Database for the NYSE 1815 to 1925: Performance and Predictability, Journal of Financial Markets, 4, 1 - 32.

[26] Griffin, John, M., Xiuqing Ji, and J. Spencer Martin, 2005, Global momentum strategies: A portfolio perspective, Journal of Portfolio Management, 31, 23 - 29

[27] Grinblatt, Mark, and Bing Han, 2005, Prospect Theory, Mential Accounting, and Momentum, Journal of Financial Economics, 78, 311 - 149.

[28] Grinblatt, Mark, and Matti Keloharju, 2001, What makes investor trade? Journal of Finance 56, 589 - 616.

[29] Grinblatt, Mark, and Tobias Moskowitz, 1999, Do industries explain momentum?, Journal of Finance 54, 1249 - 1290. 
[30] Grinblatt, Mark, and Tobias Moskowitz, 2004, Predicting stock price movements from past returns: the role of consistency and tax-loss selling, Journal of Financial Economics, 71, $541-579$.

[31] Grinblatt, Mark, and Sheridan Titman, 1989, Mutual Fund Performance: An Analysis of Quarterly Portfolio Holdings, Journal of Business 62, 393 - 416.

[32] Grundy, Bruce D., and J. Spencer Martin, 2001, Understanding the nature of the risks and the source of the rewards to momentum investing, Review of Financial Studies 14, 29 78.

[33] Hong, Harrison, Terence Lim, and Jeremy C. Stein, 2000, Bad news travels slowly: Size, analyst coverage, and the profitability of momentum strategies, Journal of Finance 55, $266-295$.

[34] Hong, Harrison, and Jeremy C. Stein, 1999, A unified theory of underreaction, momentum trading and overreaction in asset markets, Journal of Finance 54, 2143 - 2184.

[35] Hou, Kewei, 2001, Information diffusion and asymmetric cross-autocorrelations in stock returns, Working Paper, The Ohio State University.

[36] Jegadeesh, Narasimhan, 1990, Evidence of predictable behavior of security returns, Journal of Finance 45, 881 - 898.

[37] Jegadeesh, Narasimhan and Sheridan Titman, 1991, Short horizon return reversals and the bid-ask spread, Working paper UCLA.

[38] Jegadeesh, Narasimhan, and Sheridan Titman, 1993, Returns to buying winners and selling losers: Implications for stock market efficiency, Journal of Finance 48, 65 - 91.

[39] Jegadeesh, Narasimhan, and Sheridan Titman, 1995, Overreaction, delayed reaction, and contrarian profits, Review of Financial Studies 8, 973 - 993.

[40] Jegadeesh, Narasimhan, and Sheridan Titman, 2001, Profitability of momentum strategies: An evaluation of alternative explanations, Journal of Finance 56, 699 - 720.

[41] Jegadeesh, Narasimhan, and Sheridan Titman, 2002, Cross-sectional and time-series determinants of momentum returns, Review of Financial Studies 15, 143 - 158.

[42] Jegadeesh, Narasimhan, and Sheridan Titman, 2005, Momentum: A review, in Advances in Behavioral Finance II, Ed. Richard Thaler, Princeton University Press, NJ.

[43] Jensen, Michael and George Bennington, 1970, Random walks and technical theories: Some additional evidence, Journal of Finance 25, 469 - 482. 
[44] Johnston, Louis D. and Samuel H. Williamson, 2008, What Was the U.S. GDP Then? Measuring Worth.

[45] Korajczyk, Robert A. and Ronnie Sadka, 2004, Are Momentum Profits Robust to Trading Costs?, Journal of Finance 59, 1039 - 1082.

[46] Lakonishok, J., R. Shleifer, and R. Vishny, 1994, Contrarian investment, extrapolation, and risk, Journal of Finance 49, 1541 - 1578.

[47] Lesmond, David, Michael Schill and Chungsheng Zhou, 2004, The Illusory Nature of Momentum Profits, Journal of Financial Economics, 71, 349 - 380.

[48] Levy, Robert, 1967, Relative strength as a criterion for investment selection, Journal of Finance, 22, 595 - 610.

[49] Lo, Andrew W., and A. Craig MacKinlay, 1990, When are contrarian profits due to stock market-overreaction? Review of Financial Studies 3, 175 - 208.

[51] Newey, W., and K. West, 1987, A Simple, Positive Semi-Definite, Heteroskedasticity and Autocorrelation Consistent Covariance Matrix, Econometrica, 55, 703-708.

[52] Officer, Lawrence H., 2008a, What Was the U.K. GDP Then?" Measuring Worth.

[53] Officer, Lawrence H., 2008b, Five Ways to Compute the Relative Value of a UK Pound Amount, 1830 to Present, Measuring Worth.

[54] Rouwenhorst, K. Geert, 1998, International momentum strategies, Journal of Finance $53,267-284$.

[55] Roll, Richard, 1983, Vas first Das? Journal of Portfolio Management, 18 - 28.

[56] Sagi, Jacob S., and Mark S. Seasholes (2007), Firm-Specific Attributes and the CrossSection of Momentum, Journal of Financial Economics 84, 389 - 434.

[57] Shleifer, A. and Vishny, R.W. (1997), The Limits of Arbitrage, Journal of Finance, 52, $35-56$.

[58] Thomas and Hwang, The 52 week high and momentum investing, Journal of Finance, 59, 2145-2176. 


\section{Appendices}

\section{A. The London Stock Market Data: 1866-1907}

The closing bid and ask prices were collected from the quotation list of The Money Market Review, a weekly financial paper published in London between 1860 and 1908. Published on Saturdays, The Money Market Review reprinted H.H. Wetenhall's official quotation list of the previous Friday's closing prices. These were the official prices published by the Committee of the Stock Exchange under the name "Course of the Exchange". The data was sampled every 28-days rather than the more traditional end of month observations, due to the newspaper's weekly publication schedule.

The official list was organized by industry and asset type. The list begins with British government debt, then lists foreign government debt, British, Commonwealth and foreign railroads and concludes with commercial securities organized by industry (banks, breweries, canals \& docks, insurance, iron coal \& steel, gas, mining, shipping, spinning, waterworks, tea, land, financial \& investment trusts and miscellaneous securities). The Money Market Review's list is not complete. Some industries do not appear on certain dates and within industries individual stocks may have no price for one or more dates. When possible, we filled in the missing price data with The Economist's "Stock Market Prices Current". The Economist 's price list included only the largest and most active securities listed in London. Since its coverage was sparse, we only employ the Economist to fill in data that was missing from the The Money Market Review. If a section of the official list was omitted from a given Money Market Review, we attempted to replace the missing data with quotes from the Economist.

The data set contains 610,421 bid and ask prices. To minimize entry time and assure quality, the data was double entered by undergraduate research assistants. As a consequence of our data entry strategy, a stock must appear on the official list for at least one January before it is included in the data set.

The official lists did not differentiate between equity and debt. Today, one would assume that an economist and trained historian would have no problem distinguishing a stock from a bond, but in the $19^{\text {th }}$ Century the difference between debt and equity was seldom obvious. The $19^{\text {th }}$ Century English publications generally referred to both debt and equity claims as "stocks". A careful examination of the claims each class of shareholder enjoyed, usually allowed us to determine if a given security was a debt or equity claim. When selecting which securities to include in our data set, we excluded all securities with fixed interest rates, a face value to be returned at a maturity date or other obvious characteristics of bonds.

In general, London securities were divided into the following types of asset classes: "stocks", "shares", "ordinary", "common", "limited", "deferred", "preference", "debenture" and "convertible" shares. Whether, the name of the share corresponded to what a modern investor would consider equity depended upon the type of company in question. We looked at each potential security and excluded every security with characteristics similar to modern day debt. "Common", "limited", and "ordinary" shares were almost always the residual claimants and therefore correspond to modern day equity. "Stock" on the other hand, was the name given to $19^{\text {th }}$ Century bonds! "Preference", "debenture" and "convertible" shares were also excluded, while "deferred" shares generally referred to debt offerings with one notable exception, the investment trusts. Many investment trusts issued only three types of shares, "preference", "debenture" and "deferred". Debenture and preference shares had a fixed dividend rate and often had a maturity date at which time the nominal amount (face value) of the share would be returned. Deferred shares in investment trusts, on the other hand, were generally the residual claimant to all income in excess of the debenture and preference obligations. We include the deferred shares of investment trusts in our data, provided the trust has no ordinary shares and the deferred shares satisfy our conditions of no maturity date and no cap on dividends.

For each January, a list of all securities was compiled, meeting our definition of equity. This list of security names and copies of the subsequent year's quotation lists, were distributed to research assistants. To eliminate typos each date was double entered by different research assistants and then compared. Therefore, to appear in the data set, a stock had to appear on the official quotation list for at least one January. 
In addition to the closing prices, we collected dividend payments and shares outstanding, for each security. These allow us to compute market values and 28-day holding period returns that accurately reflect dividend payments and stock splits. In total, the data set consists of 610,421 bid and ask prices and 39,090 dividend payments. The dividend payments were collected from the security lists of The Investor's Monthly Manual ${ }^{18}$. The Investor's Monthly Manual (IMM) published the monthly closing price, shares outstanding and last four dividends of each security, listed on the London Stock Exchange. We use the IMM to collect dividend and share histories for each security that appears in our data set. Like The Money Market Review, certain securities vanish from the IMM and reappear at a later date without explanation.

\section{A1. Capital Calls and Returns}

We use the price and dividend data to compute the 28-day holding period return for each consecutive price observation. The 28-day holding period gross return is defined as $\left(P_{t+1}+d_{t+1}\right) / P_{t}$ where $P_{t+1}$ and $P_{t}$ are the average of the bid and ask prices at time $t+1$ and $t$ respectively, and $d_{t}$ is the net dividend payments and capital calls (if any) that occurred between time $t$ and $t+1$. Capital calls are a form of reverse dividend common to $19^{\text {th }}$ Century stock exchanges.

Many $19^{\text {th }}$ Century companies issued shares with a nominal value known as the "amount". The company typically did not require the shareholders to pay for the entire share at the time of issue. Instead, shares were issued with a "par" or "paid" value that was less than the nominal amount of the share. Dividends were based on the par value of the share and not the nominal amount. For example, if a company with a $£ 100$ share with $£ 50$ paid announced a $10 \%$ declared dividend, this would amount to $£ 5$ rather than $£ 10$ :

The shareholder was legally obligated to pay the remaining capital (the difference between the nominal and paid amount) at the whim of the company. Thus the company could "call" upon its shareholders to pay for the remaining value of their shares. This call was apparently binding, as the shares in many bankrupt companies with par values less than nominal amounts traded at negative values when the implicit short put option embedded in the shares was worth more than the company's equity. ${ }^{19}$ To compute holding period returns, we treat capital calls as a negative dividend paid at the beginning of the holding period.

\section{A2. Data Limitations}

Historical data provides both a new laboratory to evaluate theories as well as a unique set of challenges. Missing observations are far more common in historical data. There are three types of missing data. Firstly, there are securities that vanish because the company goes out of business. Secondly, there are securities that are not quoted for a period and then re-appear without explanation. Finally, there are dates when the newspapers omit an entire industry from the price lists.

The first case is the well known problem of survivorship bias. Securities do not vanish from the price list at random. Instead, the companies with poor returns and low stock prices are far more likely to go bankrupt and vanish. Two characteristics of our data mitigate the potential survivorship bias. To begin with, very few securities simply "vanish". When a security disappears from our price list we generally observe its decline to zero (or negative) price before it is removed from the list. When a security vanishes we make every attempt to discover what happened to the company and compute the final holding period return. If the security vanished due to reorganization, liquidation or merger, the IMM often lists the details and we correct the last return to reflect these events.

The second and third cases of missing data are more troublesome. These are cases of securities that do not go out of business, but are merely omitted from the price list on a given day. If prices are missing completely at random, the analysis that follows can easily be altered to accommodate the missing observations. If the probability

\footnotetext{
${ }^{18}$ The IMM is available online at $\mathrm{http} / / / \mathrm{som} . y a l e . e d u / \mathrm{imm} / \mathrm{html} / \mathrm{index} \cdot \mathrm{shtml}$

${ }^{19} \mathrm{We}$ only include stocks with positive values in the analysis that follows.
} 
of observing a given return is correlated with the value of that return, however, the missing values will bias our estimates.

In the computations to follow, we form our momentum portfolios using actual prices available to Victorian era investors. We base all buy and sell decisions on actual price data. Once a portfolio is formed, however, we compute its post-formation return from observable prices if possible and interpolate data if necessary.

When necessary, we replace missing prices with interpolated data via the following algorithm. If a security vanishes from the quotation list we look ahead to the next date that a quotation is available. If the security reappears $\mathrm{N}$-periods in the future, we compute the N-period gross return and convert it to a 1-period return by taking the gross return to the $(1 / \mathrm{N})$ th power. We replace all missing 1-period returns with this interpolated return. For example, if a security's price is last observed at time $t$ and reappears $20 \%$ higher at time $t+5$ we replace the missing values at time $\mathrm{t}+1$ to $\mathrm{t}+5$ with $(1.2)^{(1 / 5)}$ : When computing multiperiod returns we assume all dividends are paid at the end of the period.

If a stock vanishes and does not reappear for one year we set its return to $-99.99 \%$. Setting all extinct stocks to $-99.99 \%$ return surely understates the true return. However, the nature of the conclusions we obtain do not change if we relax our assumptions by either ignoring the extinction (don't set the last return to $99.99 \%$ ) or ignoring all missing data by assuming it is missing at random and computing results from observable data only. Setting extinct stocks to $-99.99 \%$ obviously lowers gross returns but the relative comparisons of portfolios sorted by size or momentum are robust to the treatment of missing data - as will be discussed in detail later. Finally, it should be noted that we do not have enough industries during the Victorian age to examining the importance of industry momentum identified by Grinblatt and Moskowitz (1999).

\section{B. Duration Analyses and Tests}

\section{B1. Nonparametric tests}

Duration spells of up and down momentum cycles are tested for equality across the Victorian and CRSP eras in Table 16. The test statistics are: are (a) the Mann-Whitney-Wilcoxon (MWW) test, (b) KolmogorovSmirnov test, (c) Cramer-von Mises test, and (d) Kuiper test Entries to the table are p-values for the null hypothesis that the duration spells during the Victorian and CRSP eras are drawn from the same population. We provide a short description of the tests in this section.

The MWW test is for chances of obtaining greater observations in one population versus the other. The null hypothesis in the Mann-Whitney test is that the two samples are drawn from a single population, and therefore that their probability distributions are equal. The alternative hypothesis is that one sample is stochastically greater. It requires the two samples to be independent. In a most general formulation, the test may be thought of as testing the null hypothesis that the probability of an observation from one population $(X)$ exceeding an observation from the second population $(Y)$ is $0.5: P(X>Y)=0.5$ versus $P(X>Y)>0.5, P(X>Y)<0.5$, or $P(X>Y) \neq 0.5$ in an alternative hypothesis. The test involves the calculation of a statistic, usually called $U$ (U-statistic is due to Hoeffding (1948)) whose distribution under the null hypothesis is known - in samples of moderate size it is normally distributed.

While the MWW is based on ranks, the next statistic, the Kolmogorov-Smirnov test (K-S test) is a form of minimum distance estimation used in our case to compare two samples (two-sample $\mathrm{K}-\mathrm{S}$ test). The KolmogorovSmirnov statistic quantifies a distance between the empirical distribution functions of two samples - in our case duration spells across two eras. It has a non-standard asymptotic distribution based on a Brownian bridge. The Cramer-von Mises test is also based on distance measures between empirical (duration) distributions, yet it involves a different metric - namely a $L^{2}$ rather than Sup norm in the case of the K-S test. Finally the Kuiper test is also closely related to the K-S test but distinguishes the maximum deviation above from the one below of the two cumulative distributions being compared. 


\section{B2. Parametric Analysis}

We explain how the estimates and the tests reported in Tables 17 through 19 are computed. Let $T$ denote a random variable of duration for positive or negative momentum profits. For Table 17, let $f(t, \theta)$ denote the probability density function of $T$ with the parameter (vector) $\theta$. For the exponential distribution, $f(t, \lambda)=\lambda e^{-\lambda t}$. For the Gamma distribution, $f(t, \lambda, \kappa)=\frac{(t \lambda)^{\kappa-1} \lambda e^{-\lambda t}}{\Gamma(\kappa)}$. And, for the Weibull distribution, $f(t, \lambda, p)=\lambda p(\lambda t)^{p-1} e^{-(\lambda t)^{p}}$. We can use the maximum likelihood estimation of parameters of interest.

Given a sample of duration of $\left\{t_{n}\right\}_{n=1}^{T}$, we compute the log likelihood of sample as $\mathcal{L}(\theta)=\sum_{n=1}^{T} \log \left(f\left(t_{n}, \theta\right)\right)$. Then, the ML estimates of $\theta$ are computed from

$$
\theta_{M L}=\operatorname{argmax}_{\theta} \mathcal{L}(\theta) \text {. }
$$

Let $d$ denote the dimension of $\theta$. For the exponential distribution, $d=1$, for the Gamma and the Weibull distributaion, $d=2$. Standard errors of $\theta_{M L}$ are obtained from the diagonal elements of $\mathcal{J}(\theta)^{-1}$. The (i,j) element of $\mathcal{J}(\theta)$ is

which is numerically approximated as:

$$
\mathcal{J}(\theta)_{i, j}=-E\left[\frac{\partial^{2}}{\partial \theta_{i} \partial \theta_{j}} \log (f(T, \theta)) \mid \theta\right],
$$

$$
-\frac{1}{T} \frac{\frac{\mathcal{L}\left(\theta_{M L}+\varepsilon l_{\mathrm{i}}+\varepsilon \mathrm{l}_{\mathrm{j}}\right)-\mathcal{L}\left(\theta_{M L}-\varepsilon \mathrm{l}_{\mathrm{i}}+\varepsilon \mathrm{l}_{\mathrm{j}}\right)}{2 \varepsilon}-\frac{\mathcal{L}\left(\theta_{M L}+\varepsilon \mathrm{l}_{\mathrm{i}}-\varepsilon \mathrm{l}_{\mathrm{j}}\right)-\mathcal{L}\left(\theta_{M L}-\varepsilon \mathrm{l}_{\mathrm{i}}-\varepsilon \mathrm{l}_{\mathrm{j}}\right)}{2 \varepsilon}}{2 \varepsilon},
$$

where $\varepsilon$ is set as $10^{-6}$ and $\mathrm{l}_{\mathrm{i}}$ is a $d$-dimensional vector of zero except $\mathrm{i}$-th component of 1 . After approximating the $d$-by- $d$ matrix of $\mathcal{J}(\theta)$ as the above, we take the diagonal elements of the inverse matrix of $T \cdot \overline{\mathcal{J}(\theta)}-1$ to get the standard errors of $\theta_{M L}$. For Table 18, we first apply a 3 month MA filter to all the data, including momentum profits and calculate momentum durations and the corresponding state variables.

Next, we keep duration data above 3 months and we estimate parameters and standard errors of an accelerated failure time (AFT) model which takes the form

$$
T=T_{0} \exp (X \beta)
$$

where $T$ is the duration, $T_{0}$ is the baseline failure time which follows the baseline distribution, $X$ is a vector of explanatory variables, and $\beta$ is the parameter vector. We assume $T_{0}$ follows the Gamma distribution or the Weibull distribution with $\lambda=1$. Let $f(t, x, \theta)$ denote the density of duration $t$ with the state variable of $x$. Under the Gamma distribution, the density of $t$ with explanatory variables of $x$ is

And, under the Weibull distribution, that is

$$
f(t, x, \beta, \kappa)=\frac{(t \lambda)^{\kappa-1} \lambda e^{-\lambda t}}{\Gamma(\kappa)}
$$

$$
f(t, x, \beta, p)=\lambda p(\lambda t)^{p-1} e^{-(\lambda t)^{p}} .
$$

Here, we set $\lambda^{-1}=\exp \left(\beta_{0}+\beta_{1} r_{f}+\beta_{2} r_{\text {stock }}\right)$. Note that the dimension of $\theta$ is $d=4$ with our baseline model and the specification of AFT. Given a sample of duration of $\left\{t_{n}\right\}_{n=1}^{T}$ and state variables of $\left\{x_{n}\right\}_{n=1}^{T}$, we compute the $\log$ likelihood of sample as $\mathcal{L}(\theta)=\sum_{n=1}^{T} \log \left(f\left(t_{n}, x_{n}, \theta\right)\right)$. We use again ML to estimate the models.

From the estimates of $\beta_{1}$ for Victorian era with a Gamma distribution, we can infer that a $1 \%$ increase in the level of $r_{f}$ will induce $40 \%$ increase of the average duration time. Table 19 shows the difference of the effects of state variables across UP and DOWN cycles. To test this formally, we pool the duration data as $\left\{t_{n}, d d_{n}\right\}_{n=1}^{T}$ for each era. $d d_{n}$ is a down dummy variable which is 1 if the cycle is down and 0 otherwise. Under the Gamma distribution, the density of $t$ with explanatory variables of $x$ and the down dummy $d d$ is

$$
f\left(t, d d, x, \beta, \kappa, \beta_{d d}, \kappa_{d d}\right)=\frac{(t \lambda)^{\kappa^{*}-1} \lambda e^{-\lambda t}}{\Gamma\left(\kappa^{*}\right)} .
$$

And, under the Weibull distribution, that is

$$
f\left(t, d d, x, \beta, p, \beta_{d d}, p_{d d}\right)=\lambda p^{*}(\lambda t)^{p^{*}-1} e^{-(\lambda t)^{p^{*}}} .
$$


Here, we set $\lambda^{-1}=\exp \left(\beta_{0}+\beta_{1} r_{f}+\beta_{2} r_{\text {stock }}+d d\left(\beta_{0, d d}+\beta_{1, d d} r_{f}+\beta_{2, d d} r_{\text {stock }}\right)\right), \kappa^{*}=\kappa+d d \kappa_{d d}$ and $p^{*}=p+d d p_{d d}$. Note that the dimension of $\theta$ is $d=4$ under given parametric assumptions and the specification of AFT. We use the same method as in table 18 for estimating parameters and the standard errors of those. The results of Table 19 show that we can reject the null that the effect of risk free rate is the same across UP and DOWN cycles. 


\section{Table 1: Returns of Relative Strength Portfolios - Short horizons London Stock Exchange 1866-1907}

The relative strength portfolios are formed on J-month lagged returns and held for $\mathrm{K}$ months. The values of $\mathrm{J}$ and $\mathrm{K}$ for the different strategies are indicated in the first column and row respectively. The stocks are ranked in ascending order on the basis of J-month lagged returns. A value-weighted portfolio of stocks in the lowest 33rd percentile past return is the sell portfolio. A value-weighted portfolio of stocks in the highest 67 th percentile past return is the buy portfolio. t-statistics are between parentheses. As noted at the beginning of section 2, we considered 28-day periods as months in our calculations. Moreover, when considering a year, i.e. $\mathrm{K}=12$ in Jegadeesh and Titman, we used 13 28-day periods.

\begin{tabular}{|c|c|c|c|c|c|c|}
\hline \multirow{2}{*}{$\mathrm{J}$} & & \multirow{2}{*}{$\begin{array}{l}\text { skip } \\
\text { month }\end{array}$} & \multicolumn{4}{|c|}{ K } \\
\hline & & & 3 & 6 & 9 & 13 \\
\hline 3 & Buy & $\begin{array}{c}-0.0046 \\
(-2.6500)\end{array}$ & $\begin{array}{c}0.0001 \\
(0.0020)\end{array}$ & $\begin{array}{c}0.0016 \\
(1.4428)\end{array}$ & $\begin{array}{c}0.0019 \\
(1.8167)\end{array}$ & $\begin{array}{c}0.0023 \\
(2.4009)\end{array}$ \\
\hline 3 & Sell & $\begin{array}{c}0.0020 \\
(1.4300)\end{array}$ & $\begin{array}{c}0.0000 \\
(0.0001)\end{array}$ & $\begin{array}{c}-0.0003 \\
(-0.2643)\end{array}$ & $\begin{array}{c}0.0000 \\
(0.0389)\end{array}$ & $\begin{array}{c}0.0001 \\
(0.1415)\end{array}$ \\
\hline 3 & Buy-Sell & $\begin{array}{c}-0.0066 \\
(-3.3800)\end{array}$ & $\begin{array}{c}0.0001 \\
(0.0019)\end{array}$ & $\begin{array}{c}0.0019 \\
(1.8938)\end{array}$ & $\begin{array}{c}0.0018 \\
(2.1452)\end{array}$ & $\begin{array}{c}0.0022 \\
(3.0538)\end{array}$ \\
\hline 6 & Buy & $\begin{array}{c}-0.0017 \\
(-1.1800)\end{array}$ & $\begin{array}{c}0.0018 \\
(1.5419)\end{array}$ & $\begin{array}{c}0.0026 \\
(2.3060)\end{array}$ & $\begin{array}{c}0.0030 \\
(2.8644)\end{array}$ & $\begin{array}{c}0.0032 \\
(3.2670)\end{array}$ \\
\hline 6 & Sell & $\begin{array}{c}0.0009 \\
(0.6000)\end{array}$ & $\begin{array}{c}-0.0008 \\
(-0.6585)\end{array}$ & $\begin{array}{c}-0.0008 \\
(-0.6817)\end{array}$ & $\begin{array}{c}-0.0007 \\
(-0.6093)\end{array}$ & $\begin{array}{c}0.0000 \\
(-0.0161)\end{array}$ \\
\hline 6 & Buy-Sell & $\begin{array}{c}-0.0026 \\
(-1.4800)\end{array}$ & $\begin{array}{c}0.0027 \\
(2.0877)\end{array}$ & $\begin{array}{c}0.0034 \\
(2.9693)\end{array}$ & $\begin{array}{c}0.0038 \\
(3.6612)\end{array}$ & $\begin{array}{c}0.0032 \\
(3.6469)\end{array}$ \\
\hline 9 & Buy & $\begin{array}{c}-0.0004 \\
(-0.3100)\end{array}$ & $\begin{array}{c}0.0026 \\
(2.1190)\end{array}$ & $\begin{array}{c}0.0035 \\
(3.0610)\end{array}$ & $\begin{array}{c}0.0034 \\
(3.2159)\end{array}$ & $\begin{array}{c}0.0034 \\
(3.4521)\end{array}$ \\
\hline 9 & Sell & $\begin{array}{c}0.0000 \\
(-0.0200)\end{array}$ & $\begin{array}{c}-0.0023 \\
(-1.3453)\end{array}$ & $\begin{array}{c}-0.0019 \\
(-1.2881)\end{array}$ & $\begin{array}{l}-0.0013 \\
(-0.983)\end{array}$ & $\begin{array}{c}-0.0006 \\
(-0.4663)\end{array}$ \\
\hline 9 & Buy-Sell & $\begin{array}{c}-0.0004 \\
(-0.2500)\end{array}$ & $\begin{array}{c}0.0049 \\
(2.8105)\end{array}$ & $\begin{array}{c}0.0054 \\
(3.7652)\end{array}$ & $\begin{array}{c}0.0048 \\
(3.7538)\end{array}$ & $\begin{array}{c}0.0040 \\
(3.7212)\end{array}$ \\
\hline 13 & Buy & $\begin{array}{c}0.0022 \\
(1.7800)\end{array}$ & $\begin{array}{c}0.0032 \\
(2.8363)\end{array}$ & $\begin{array}{c}0.0034 \\
(3.1544)\end{array}$ & $\begin{array}{c}0.0034 \\
(3.2221)\end{array}$ & $\begin{array}{c}0.0033 \\
(3.3945)\end{array}$ \\
\hline 13 & Sell & $\begin{array}{c}-0.0016 \\
(-0.9600)\end{array}$ & $\begin{array}{c}-0.0023 \\
(-1.3502)\end{array}$ & $\begin{array}{c}-0.0014 \\
(-0.9738)\end{array}$ & $\begin{array}{c}-0.0007 \\
(-0.4948)\end{array}$ & $\begin{array}{c}-0.0001 \\
(-0.0540)\end{array}$ \\
\hline 13 & Buy-Sell & $\begin{array}{c}0.0038 \\
(2.2100)\end{array}$ & $\begin{array}{c}0.0055 \\
(3.3047)\end{array}$ & $\begin{array}{c}0.0048 \\
(3.4207)\end{array}$ & $\begin{array}{c}0.0040 \\
(3.2261)\end{array}$ & $\begin{array}{c}0.0034 \\
(3.0743)\end{array}$ \\
\hline
\end{tabular}




\section{Table 2: Betas and Market Capitalization of Relative Strength Portfolios London Stock Exchange 1866-1907}

The relative strength portfolios are formed on a 6-month lagged returns and held for 6 months. The stocks are ranked in ascending order on the basis of 6-month lagged returns. A value-weighted portfolio of stocks in the lowest 33rd percentile past return is the sell portfolio. A value-weighted portfolio of stocks in the highest 67 th percentile past return is the buy portfolio. The remaining are assigned in the middle portfolio.

\begin{tabular}{lcc}
\hline CAPM Beta & $\begin{array}{c}\text { Average Market Cap } \\
\text { (million pounds) }\end{array}$ \\
\hline Buy & 1.171 & 13.369 \\
Middle 1/3 & 0.735 & 19.409 \\
Sell & 1.195 & 12.437 \\
& & \\
Buy-Sell & -0.024 & \\
\hline
\end{tabular}




\section{Table 3: Monthly Returns of Size-based and Beta-based Relative Strength Portfolios London Stock Exchange 1866-1907}

The relative strength portfolios are formed on a 6-month lagged returns and held for 6 months. The stocks are ranked in ascending order on the basis of 6-month lagged returns. A value-weighted portfolio of stocks in the lowest 33rd percentile past return is the sell portfolio and A value-weighted portfolio of stocks in the highest 67 th percentile past return is the buy portfolio. The remaining are assigned in the middle portfolio. Average monthly returns and excess returns of these portfolios and the returns of the relative strength portfolios formed using size-based and beta-based subsamples of securities are reported here. Size is sorted in lower, middle and highest thirds. Panel A reports average returns. Panel B reports Jensen's CAPM alphas. And, Panel C reports CAPM regressions augmented with a NBER Business Cycle dummy.

\begin{tabular}{|c|c|c|c|c|}
\hline Past Returns & All & Small & Medium & Large \\
\hline \multicolumn{5}{|c|}{ Panel A: Size-sorted Average returns and t-stats } \\
\hline Buy & $\begin{array}{c}0.0026 \\
(2.3060)\end{array}$ & $\begin{array}{c}0.0014 \\
(0.7968)\end{array}$ & $\begin{array}{c}0.0034 \\
(2.7908)\end{array}$ & $\begin{array}{c}0.0024 \\
(2.0136)\end{array}$ \\
\hline Middle & $\begin{array}{c}0.0020 \\
(3.0078)\end{array}$ & $\begin{array}{l}-0.0018 \\
(-1.042)\end{array}$ & $\begin{array}{c}0.0006 \\
(0.4981)\end{array}$ & $\begin{array}{c}0.0021 \\
(3.1300)\end{array}$ \\
\hline Sell & $\begin{array}{c}-0.0008 \\
(-0.6817)\end{array}$ & $\begin{array}{c}-0.0019 \\
(-1.0100)\end{array}$ & $\begin{array}{c}-0.0019 \\
(-1.4414)\end{array}$ & $\begin{array}{c}-0.0007 \\
(-0.5188)\end{array}$ \\
\hline Buy-Sell & $\begin{array}{c}0.0034 \\
(2.9693)\end{array}$ & $\begin{array}{c}0.0033 \\
(2.7130)\end{array}$ & $\begin{array}{c}0.0053 \\
(6.0779)\end{array}$ & $\begin{array}{c}0.0031 \\
(2.4098)\end{array}$ \\
\hline \multicolumn{5}{|c|}{ Panel B: Excess returns (Jensen's CAPM alphas) } \\
\hline Buy & $\begin{array}{c}-0.0040 \\
(-5.0428)\end{array}$ & $\begin{array}{l}-0.0028 \\
(-1.5862)\end{array}$ & $\begin{array}{c}-0.0013 \\
(-1.1498)\end{array}$ & $\begin{array}{c}-0.0044 \\
(-4.9347)\end{array}$ \\
\hline Middle & $\begin{array}{c}-0.0029 \\
(-6.6399)\end{array}$ & $\begin{array}{c}-0.0052 \\
(-2.8756)\end{array}$ & $\begin{array}{c}-0.0032 \\
(-2.6764)\end{array}$ & $\begin{array}{c}-0.0029 \\
(-6.6667)\end{array}$ \\
\hline Sell & $\begin{array}{c}-0.0075 \\
(-8.1057)\end{array}$ & $\begin{array}{c}-0.0071 \\
(-3.7656)\end{array}$ & $\begin{array}{l}-0.0074 \\
(-6.241)\end{array}$ & $\begin{array}{l}-0.0075 \\
(-7.486)\end{array}$ \\
\hline Buy-Sell & $\begin{array}{c}0.0035 \\
(2.9840) \\
\end{array}$ & $\begin{array}{c}0.0043 \\
(3.4295) \\
\end{array}$ & $\begin{array}{c}0.0061 \\
(6.8496) \\
\end{array}$ & $\begin{array}{c}0.0031 \\
(2.3639) \\
\end{array}$ \\
\hline
\end{tabular}

Table continued on next page ... 
Table 3 Continued

\begin{tabular}{|c|c|c|c|c|c|c|c|c|}
\hline \multicolumn{9}{|c|}{ Panel C: Jensen Alphas with Market and NBER Business Cycle Dummy } \\
\hline Past Returns & All & Small & Medium & Large & All & Small & Medium & Large \\
\hline & \multicolumn{4}{|c|}{ Jensen Alphas } & \multicolumn{4}{|c|}{$\begin{array}{l}\text { Beta on NBER Business Cycle Dummy } \\
\text { (Dummy } 1 \text { if recession) }\end{array}$} \\
\hline Buy & $\begin{array}{c}-0.0041 \\
(-3.6934)\end{array}$ & $\begin{array}{c}-0.001 \\
(-0.3983)\end{array}$ & $\begin{array}{c}-0.0002 \\
(-0.1022)\end{array}$ & $\begin{array}{c}-0.0046 \\
(-3.6858)\end{array}$ & $\begin{array}{c}0.0002 \\
(0.1573)\end{array}$ & $\begin{array}{c}-0.0036 \\
(-1.0369)\end{array}$ & $\begin{array}{c}-0.0023 \\
(-1.0167)\end{array}$ & $\begin{array}{c}0.0004 \\
(0.2553)\end{array}$ \\
\hline Middle & $\begin{array}{c}-0.0028 \\
(-4.4469)\end{array}$ & $\begin{array}{c}-0.0037 \\
(-1.4656)\end{array}$ & $\begin{array}{c}-0.0019 \\
(-1.1618)\end{array}$ & $\begin{array}{c}-0.0028 \\
(-4.5684)\end{array}$ & $\begin{array}{c}-0.0003 \\
(-0.3852)\end{array}$ & $\begin{array}{c}-0.0029 \\
(-0.8222)\end{array}$ & $\begin{array}{c}-0.0025 \\
(-1.0538)\end{array}$ & $\begin{array}{c}-0.0002 \\
(-0.2391)\end{array}$ \\
\hline Sell & $\begin{array}{c}-0.0074 \\
(-5.6204)\end{array}$ & $\begin{array}{c}-0.0058 \\
(-2.2076)\end{array}$ & $\begin{array}{c}-0.0048 \\
(-2.8974)\end{array}$ & $\begin{array}{c}-0.0077 \\
(-5.4264)\end{array}$ & $\begin{array}{c}-0.0004 \\
(-0.1968)\end{array}$ & $\begin{array}{c}-0.0025 \\
(-0.6662)\end{array}$ & $\begin{array}{c}-0.0052 \\
(-2.2123)\end{array}$ & $\begin{array}{c}0.0003 \\
(0.1533)\end{array}$ \\
\hline Buy-Sell & $\begin{array}{c}0.0032 \\
(1.9362)\end{array}$ & $\begin{array}{c}0.0048 \\
(2.7614)\end{array}$ & $\begin{array}{c}0.0047 \\
(3.7368)\end{array}$ & $\begin{array}{c}0.0031 \\
(1.6406)\end{array}$ & $\begin{array}{l}6.08 \mathrm{E}-04 \\
(0.2613)\end{array}$ & $\begin{array}{c}-0.0011 \\
(-0.4610)\end{array}$ & $\begin{array}{c}0.0028 \\
(1.6222)\end{array}$ & $\begin{array}{l}1.44 \mathrm{E}-04 \\
(0.0551)\end{array}$ \\
\hline
\end{tabular}




\section{Table 4: Sensitivity to treatment of exit firms - CAPM Alphas}

In this table we relax the assumption that extinct stocks have $-99.99 \%$ return. The relative strength portfolios are formed on a 6month lagged returns and held for 6 months. The stocks are ranked in ascending order on the basis of 6-month lagged returns. A value-weighted portfolio of stocks in the lowest 33rd percentile past return is the sell portfolio and A value-weighted portfolio of stocks in the highest 67 th percentile past return is the buy portfolio. The remaining are assigned in the middle portfolio. Average monthly returns and excess returns of these portfolios and the returns of the relative strength portfolios formed using size-based and beta-based subsamples of securities are reported here. Size is sorted in lower, middle and highest thirds.

\begin{tabular}{lccc}
\hline & Small & Medium & Large \\
\hline \multirow{4}{*}{ Buy } & -0.0028 & -0.0013 & -0.0044 \\
\multirow{3}{*}{ Middle } & $(-1.5862)$ & $(-1.1498)$ & $(-4.9347)$ \\
& -0.0052 & -0.0032 & -0.0029 \\
Sell & $(-2.8756)$ & $(-2.6764)$ & $(-6.6667)$ \\
& -0.0071 & -0.0074 & -0.0075 \\
Buy-Sell & $(-3.7656)$ & $(-6.241)$ & $(-7.486)$ \\
& 0.0043 & 0.0061 & 0.0031 \\
& $(3.4295)$ & $(6.8496)$ & $(2.3639)$ \\
& & & \\
Buy & Panel B: Exit firms & not set at $-99.99 \%$ & \\
& 0.0024 & 0.0025 & -0.0011 \\
Middle & $(2.4802)$ & $(4.6837)$ & $(-2.3061)$ \\
& 0.0012 & 0.0008 & -0.001 \\
\multirow{2}{*}{ Sell } & $(2.1281)$ & $(2.1064)$ & $(-3.8416)$ \\
& -0.0014 & -0.0034 & -0.0048 \\
Buy-Sell & $(-1.3781)$ & $(-5.0422)$ & $(-8.3276)$ \\
& 0.0038 & 0.0059 & 0.0037 \\
& $(3.7387)$ & $(8.2087)$ & $(4.2804)$ \\
\hline
\end{tabular}




\section{Table 5: Monthly Returns on Size-based Relative Strength Portfolio by Calendar Months. London Stock Exchange 1866-1907}

The relative strength portfolios are formed on a 6-month lagged returns and held for 6 months. The stocks are ranked in ascending order on the basis of 6-month lagged returns. A value-weighted portfolio of stocks in the lowest 33rd percentile past return is the sell portfolio. A value-weighted portfolio of stocks in the highest 67 th percentile past return is the buy portfolio. Returns are 28-day, not monthly. January returns are coded as the first 28-day return entirely in the new year. P-value shows that the result of t-test on the null of equality with Jan.

\begin{tabular}{|c|c|c|c|c|}
\hline Month & All & Small & Med & Large \\
\hline \multicolumn{5}{|c|}{ Panel A: Return on Loser, Middle, and Winner Portfolio } \\
\hline & \multicolumn{4}{|c|}{ Return on 6-6 portfolios: Losers } \\
\hline Jan & 0.0020 & 0.0080 & 0.0039 & 0.0017 \\
\hline Feb-Dec & -0.0010 & -0.0027 & -0.0022 & -0.0008 \\
\hline \multirow[t]{2}{*}{ p-value } & 0.5300 & 0.1400 & 0.2200 & 0.6100 \\
\hline & \multicolumn{4}{|c|}{ Return on 6-6 portfolios: Middle } \\
\hline Jan & 0.0103 & 0.0096 & 0.0094 & 0.0105 \\
\hline Feb-Dec & 0.0014 & -0.0029 & -0.0001 & 0.0015 \\
\hline \multirow[t]{2}{*}{ p-value } & 0.0000 & 0.0700 & 0.0400 & 0.0000 \\
\hline & \multicolumn{4}{|c|}{ Return on 6-6 portfolios: Winners } \\
\hline Jan & 0.0082 & 0.0087 & 0.0132 & 0.0076 \\
\hline Feb-Dec & 0.0024 & 0.0008 & 0.0027 & 0.0023 \\
\hline p-value & 0.1700 & 0.2500 & 0.0300 & 0.2400 \\
\hline \multicolumn{5}{|c|}{ Panel B: Return on Winner - Loser Portfolio } \\
\hline Jan & 0.0063 & 0.0005 & 0.0095 & 0.0060 \\
\hline Jan & $(2.3922)$ & $(0.1770)$ & $(4.3477)$ & $(2.0312)$ \\
\hline Feb-Dec & 0.0032 & 0.0035 & 0.0050 & 0.0029 \\
\hline FEU-Ded & $(2.5891)$ & $(2.7262)$ & $(5.3517)$ & $(2.0833)$ \\
\hline p-value & 0.4694 & 0.5089 & 0.1700 & 0.5130 \\
\hline
\end{tabular}




\section{Table 6: Sensitivity to treatment of exit firms - Average returns}

We relaxed our assumptions by either ignoring the extinction (don't set the last return to $-99.99 \%$ ) or ignoring all missing data by assuming it is missing completely at random (MCAR) and computing results from observable data only. The relative strength portfolios are formed on a 6-month lagged returns and held for 6 months. The stocks are ranked in ascending order on the basis of 6-month lagged returns. A value-weighted portfolio of stocks in the lowest 33rd percentile past return is the sell portfolio and A value-weighted portfolio of stocks in the highest 67 th percentile past return is the buy portfolio. The remaining are assigned in the middle portfolio. Average monthly returns and excess returns of these portfolios and the returns of the relative strength portfolios formed using size-based and beta-based subsamples of securities are reported here. Size is sorted in lower, middle and highest thirds.

\begin{tabular}{lccc}
\hline Size & MCAR & Not set at $-99.99 \%$ & Set at $-99.99 \%$ \\
\hline \multirow{4}{*}{ Small } & Panel A: Size-sorted & Average monthly returns \\
& 0.0031 & 0.0028 & 0.0033 \\
\multirow{2}{*}{ Medium } & $(3.0173)$ & $(2.8179)$ & $(2.7130)$ \\
& 0.0049 & 0.0049 & 0.0053 \\
Large & $(6.6676)$ & $(6.8329)$ & $(6.0779)$ \\
& 0.0034 & 0.0034 & 0.0031 \\
& $(3.9647)$ & $(4.0896)$ & $(2.4098)$ \\
\hline \multirow{2}{*}{ Small } & Panel B: Excess & returns (Jensen's CAPM alphas) \\
& 0.0040 & 0.0037 & 0.0043 \\
Medium & $(4.0116)$ & $(3.7387)$ & $(3.4295)$ \\
& 0.0058 & 0.0059 & 0.0061 \\
Large & $(8.1034)$ & $(8.2087)$ & $(6.8496)$ \\
& 0.0037 & 0.0037 & 0.0031 \\
& $(4.0850)$ & $(4.2804)$ & $(2.3639)$ \\
\hline
\end{tabular}




\section{Table 7: Monthly Returns of Relative Strength Portfolios - Long horizons London Stock Exchange 1866-1907}

The relative strength portfolios are formed on J-month lagged returns and held for $\mathrm{K}$ months. The values of $\mathrm{J}$ and $\mathrm{K}$ for the different strategies are indicated in the first column and row respectively. The stocks are ranked in ascending order on the basis of J-month lagged returns. A value-weighted portfolio of stocks in the lowest 33rd percentile past return is the sell portfolio. A value-weighted portfolio of stocks in the highest 67 th percentile past return is the buy portfolio. t-statistics are between parentheses. As noted at the beginning of section 2, we considered 28-day periods as months in our calculations. Moreover, when considering a year, i.e. $\mathrm{K}=12$ in Jegadeesh and Titman, we used 13 28-day periods. It is important to note that the results are computed from all available time periods. This means the sample periods vary by column. For example the portfolio return in year 2 after formation is computed by looking at the buy-sell portfolio 14-26 months after formation, so our first "year 2" observation is July 1867-June 1868. Our first "year 5" observation doesn't begin until July 1871, however. Therefore the Year 2 column has more observations then the Year 5 column. This is also why the various columns do not add up.

\begin{tabular}{|c|c|c|c|c|c|c|c|}
\hline \multirow{2}{*}{$\mathrm{J}$} & & \multicolumn{6}{|c|}{$\mathrm{K}$} \\
\hline & & Year 2 & Year 3 & Year 4 & Year 5 & Year 2-5 & Year 3-5 \\
\hline \multirow{2}{*}{3} & \multirow{2}{*}{ Buy } & 0.0028 & 0.0028 & 0.0026 & 0.0019 & 0.0025 & 0.0024 \\
\hline & & $(3.4085)$ & $(3.4459)$ & $(3.2127)$ & $(1.8392)$ & $(3.1330)$ & $(2.9863)$ \\
\hline \multirow{2}{*}{3} & \multirow{2}{*}{ Sell } & 0.0018 & 0.0024 & 0.0028 & 0.0031 & 0.0022 & 0.0027 \\
\hline & & (1.9838) & $(2.6039)$ & $(3.1467)$ & $(3.5481)$ & $(2.4413)$ & $(3.1020)$ \\
\hline \multirow{2}{*}{3} & \multirow{2}{*}{ Buy-sell } & 0.001 & 0.0004 & -0.0002 & -0.0013 & 0.0004 & -0.0003 \\
\hline & & $(2.2551)$ & $(0.8765)$ & $(-0.4505)$ & $(-1.6807)$ & (1.1792) & $(-1.0119)$ \\
\hline \multirow{2}{*}{6} & \multirow{2}{*}{ Buy } & 0.0032 & 0.0032 & 0.0026 & 0.002 & 0.0028 & 0.0027 \\
\hline & & $(3.9071)$ & (4.1021) & (3.0710) & $(2.2091)$ & $(3.4553)$ & $(3.3621)$ \\
\hline \multirow{2}{*}{6} & \multirow{2}{*}{ Sell } & 0.0015 & 0.0022 & 0.0033 & 0.0034 & 0.0022 & 0.0029 \\
\hline & & (1.4816) & (2.1186) & $(3.3508)$ & $(3.4622)$ & (2.2689) & (2.9919) \\
\hline \multirow{2}{*}{6} & \multirow{2}{*}{ Buy-sell } & 0.0017 & 0.001 & -0.0006 & -0.0014 & 0.0006 & -0.0002 \\
\hline & & $(2.6502)$ & $(1.4971)$ & $(-1.0493)$ & $(-2.1428)$ & $(1.1576)$ & $(-0.3828)$ \\
\hline \multirow{2}{*}{9} & \multirow{2}{*}{ Buy } & 0.0033 & 0.003 & 0.0022 & 0.0021 & 0.0026 & 0.0025 \\
\hline & & $(3.8523)$ & $(3.5455)$ & $(2.4311)$ & $(2.2850)$ & (3.1731) & (2.9911) \\
\hline \multirow{2}{*}{9} & \multirow{2}{*}{ Sell } & 0.0016 & 0.0027 & 0.0035 & 0.0034 & 0.0025 & 0.0032 \\
\hline & & (1.6110) & $(2.5483)$ & $(3.4945)$ & (3.1871) & $(2.5406)$ & $(3.3295)$ \\
\hline \multirow{2}{*}{9} & \multirow{2}{*}{ Buy-sell } & 0.0016 & 0.0003 & -0.0014 & -0.0013 & 0.0001 & -0.0008 \\
\hline & & $(2.1863)$ & $(0.3738)$ & $(-1.8331)$ & $(-1.6262)$ & $(0.1324)$ & $(-1.4513)$ \\
\hline \multirow{2}{*}{13} & \multirow{2}{*}{ Buy } & 0.0035 & 0.0029 & 0.0018 & 0.0022 & 0.0026 & 0.0023 \\
\hline & & (4.0243) & $(3.4390)$ & (1.8966) & $(2.2931)$ & $(3.1640)$ & (2.6976) \\
\hline \multirow{2}{*}{13} & \multirow{2}{*}{ Sell } & 0.0019 & 0.0027 & 0.004 & 0.0033 & 0.0029 & 0.0035 \\
\hline & & $(1.7501)$ & $(2.5532)$ & $(3.7176)$ & $(2.8022)$ & $(2.7927)$ & $(3.4251)$ \\
\hline \multirow{2}{*}{13} & \multirow{2}{*}{ Buy-sell } & 0.0016 & 0.0002 & -0.0022 & -0.0012 & -0.0002 & -0.0012 \\
\hline & & (1.9642) & $(0.2816)$ & $(-2.4996)$ & $(-1.1253)$ & $(-0.3999)$ & $(-1.9273)$ \\
\hline
\end{tabular}




\section{Table 8: Reversal Equality Tests}

We form a $T \times N$ matrix $X$ where $X(t, n)$ is the holding period return from month $(t-12)$ to month $t$ on the winner minus loser portfolio formed $n \times 13$ "months" earlier. Let $\dot{X}(t, n) \equiv(X(t, n+1)-X(t, 1))$. The entries to the table report the mean return of the winner minus loser portfolios 1 through 5 years after formation. We test of the null that $E[\dot{\mathrm{X}}(t, n)]=0 \forall t$ and $n$ via a Wald test $W=g \operatorname{Cov}(g)^{-1} g$ with $g=\left(\sum_{t}[\dot{\mathrm{X}}(t, 1)] / T, \ldots, \sum_{t}[\dot{\mathrm{X}}(t, 4)] / T\right)$ and $\operatorname{Cov}(g)$ is estimated using the Newey-West procedure with 13 lags. The test statistic is under the null $W \sim \chi^{2}(4)$, asymptotically.

Panel A: Average monthly return in year $\mathrm{N}$ after formation

\begin{tabular}{|c|c|c|c|c|}
\hline 1 & 2 & 3 & 4 & 5 \\
\hline 0.0034 & 0.0016 & 0.0006 & -0.0006 & -0.0015 \\
\hline \multicolumn{5}{|c|}{ Panel B: Wald test that average monthly return is equal in years 1 through 5} \\
\hline & W-stat & & p-value & \\
\hline & 35.14 & & 0.00 & \\
\hline
\end{tabular}

Panel C: P-values for Wald test that average monthly return in $\mathrm{N}$ years after formation = average monthly return $\mathrm{J}$ years after formation for any $\mathrm{N}, \mathrm{J}$

\begin{tabular}{lccccc}
\hline & & \multicolumn{5}{c}{ N } \\
\cline { 2 - 6 } & 1 & 2 & 3 & 4 & 5 \\
\hline 1 & - & & & \\
2 & 0 & - & & \\
3 & 0 & 0.16 & - & - & \\
4 & 0 & 0.02 & 0.12 & 0.22 & - \\
5 & 0 & 0 & 0.01 & & \\
\hline
\end{tabular}




\section{Table 9: Momentum Profits and Market States}

The Table reports evidence on market states and momentum pro $t$ similar to CGH (2004). At the beginning of each month $t$, all stocks are allocated into deciles based on their lagged six-month returns (from $t-5$ to $t-1$; skipping month $t$ ). Down, Avg and Up markets were defined as follows Up = market index return over past 3 years in top 1/3 of sample Avg $=$ market index return over past 3 years in middle $1 / 3$ of sample Down = market index return over past 3 years in Bottom 1/3 of sample. Profits of the momentum portfolios (winner minus loser deciles) are cumulated across holding period months $t+1$ to $t+6$ : Reported below are the mean monthly profits and CAPM alphas.

$$
\text { CRSP Data 1929-1995 London Data 1869-1907 }
$$

Panel A: Monthly returns following 36 month UP-Markets (85\% of observed states)

$\begin{array}{lcc}\text { Mean Ret } & 0.0040 & 0.0038 \\ & (3.8000) & (4.4555) \\ \text { CAPM alpha } & 0.0053 & 0.0039\end{array}$

(4.8913) (4.5414)

Panel B: Monthly returns following 36 month Down-Markets (15\% of observed states)

$\begin{array}{lcc}\text { Mean Ret } & -0.0028 & 0.0015 \\ & (-1.1061) & (0.7713) \\ \text { CAPM alpha } & -0.0002 & 0.0016 \\ & (-0.0971) & (0.8158)\end{array}$

Panel C: Test for equality Up= Down

\begin{tabular}{lll} 
t-stat: Mean Ret & 2.5055 & 1.0474 \\
t-stat: CAPM alpha & 2.0031 & 1.0403 \\
\hline
\end{tabular}




\section{Table 10: Factor-Model Predicted Monthly Returns and Momentum London Stock Exchange 1866-1907}

All stocks are first sorted each month $t$ into quintiles based on their six-month $(t-5$ to $t$ ) predicted returns from the four-factor model:

$$
\left(r-r_{f}\right)=a+b_{1} M k t+b_{2} \text { Divyld }+b_{3} \text { Term }+b_{4} \text { Default }
$$

where excess return on market portfolio, Divyld: lagged dividend yield on market index, Term: lagged term spread (yield on British consol - yield on 30day bankbill), and Default: lagged default yield (yield on risky bonds (brit RR) - yield on British consol). This table employs the nonoverlapping-return method used by CS (2002). In Panel A, F-stat shows f-statistic on the null that $b_{2}=b_{3}=b_{4}=0$ and $R^{2}$ is for the regression of CAPM residuals on Divyld,Term, and Default. Panel B reports sorts based on predicted returns of the four factor model.

\begin{tabular}{|c|c|c|c|c|c|c|c|c|c|}
\hline \multicolumn{10}{|c|}{ Panel A: Regression Results } \\
\hline & \multicolumn{3}{|c|}{ Sell } & \multicolumn{3}{|c|}{ Mid } & \multicolumn{3}{|c|}{ Buy } \\
\hline & Small & Med & Large & Small & Med & Large & Small & Med & Large \\
\hline \multirow{2}{*}{$b_{2}$} & 0.2964 & -0.1459 & 0.174 & 0.0688 & 0.1374 & -0.0584 & 0.018 & 0.0676 & 0.0562 \\
\hline & $(0.8799)$ & $(-0.6828)$ & $(0.9542)$ & $(0.2115)$ & $(0.6439)$ & $(-0.7423)$ & $(0.0568)$ & $(0.3213)$ & $(0.3479)$ \\
\hline \multirow{2}{*}{$b_{3}$} & 0.3009 & 0.1767 & -0.2143 & 0.4298 & 0.1904 & 0.1913 & 0.1549 & 0.3812 & -0.0572 \\
\hline & $(0.6824)$ & $(0.6315)$ & $(-0.8977)$ & $(1.0088)$ & $(0.6817)$ & $(1.8565)$ & $(0.3722)$ & $(1.3838)$ & $(-0.2707)$ \\
\hline \multirow{2}{*}{$b_{4}$} & 0.5088 & 0.3196 & 0.0919 & 0.2999 & 0.3726 & 0.0705 & 0.4165 & 0.2669 & -0.0412 \\
\hline & $(3.0051)$ & $(2.9753)$ & $(1.0031)$ & $(1.8330)$ & $(3.4753)$ & $(1.7812)$ & $(2.6067)$ & $(2.5231)$ & $(-0.5078)$ \\
\hline F-stat & 4.3343 & 3.7500 & 0.5343 & 2.1365 & 5.1524 & 2.9292 & 2.6002 & 4.0728 & 0.1689 \\
\hline$p$-value & 0.0050 & 0.0110 & 0.6590 & 0.0947 & 0.0016 & 0.0332 & 0.0515 & 0.0071 & 0.9174 \\
\hline$R^{2}$ & 0.0241 & 0.0209 & 0.0030 & 0.0120 & 0.0285 & 0.0164 & 0.0146 & 0.0227 & 0.0010 \\
\hline
\end{tabular}


Table 10 Continued

\begin{tabular}{|c|c|c|c|c|c|}
\hline \multicolumn{6}{|c|}{ Panel B: Chordia and Shivakumar (2002) predicted returns sort } \\
\hline \multirow[t]{3}{*}{ Momentum Sorts: } & & Sell & Med & Buy & Buy-Sell \\
\hline & \multirow{2}{*}{$E[R]$ low } & 0.001 & 0.0028 & 0.0031 & 0.0021 \\
\hline & & $(0.6279)$ & $(3.4371)$ & $(2.1358)$ & $(1.2509)$ \\
\hline \multirow[t]{6}{*}{ Factor sorts: } & \multirow{2}{*}{$E[R]$ med } & 0.001 & 0.0026 & 0.002 & 0.001 \\
\hline & & $(1.0013)$ & $(3.9661)$ & $(1.9003)$ & $(0.9976)$ \\
\hline & \multirow{2}{*}{$E[R]$ high } & -0.0015 & 0.0013 & 0.0039 & 0.0054 \\
\hline & & $(-0.9799)$ & $(1.4754)$ & $(3.0703)$ & $(3.7516)$ \\
\hline & \multirow{2}{*}{ high-low } & -0.0025 & -0.0015 & 0.0008 & \\
\hline & & $(-1.6382)$ & $(-1.7748)$ & $(0.5686)$ & \\
\hline
\end{tabular}




\section{Table 11: Momentum Profits and the Economy London Stock Exchange 1866-1907}

The Table reports evidence on momentum profits and the state of the economy, in particular the results of the regression:

$$
r=a+b_{1} M k t+b_{2} G D P
$$

where $r$ is annual the return on the 6-6 buy portfolio minus the return on the 6-6 sell portfolio and GDP is the demeaned real per capita GDP growth. Note that in this table we examine annual returns - not monthly - as GDP is only available at an annual frequency.

\begin{tabular}{lcccc}
\hline Size & $a$ & $b_{1}$ & $b_{2}$ & Adj. $R^{2}$ \\
\hline \multirow{2}{*}{ Small } & 0.0610 & -0.3510 & -0.8704 & \multirow{2}{*}{0.1} \\
& $(3.4017)$ & $(-1.6974)$ & $(-1.2196)$ & \\
\multirow{2}{*}{ Med } & 0.078 & -0.1771 & -0.4754 & \multirow{2}{*}{0.03} \\
& $(5.9050)$ & $(-1.1626)$ & $(-0.9044)$ & \\
\multirow{2}{*}{ Large } & 0.0321 & 0.1554 & -0.803 & \multirow{2}{*}{0.01} \\
& $(2.3458)$ & $(0.9833)$ & $(-1.4718)$ & \\
\hline
\end{tabular}




\section{Table 12: Correlations among series}

The table displays full sample estimates of the correlations computed at an annual frequency between (1) returns on buy-sell small stock portfolio, (2) buy-sell medium portfolio, (3) buy-sell large stock portfolio, (4) average cross-sectional monthly standard deviation, (5) excess returns (Mkt $\left.-r_{f}\right)$, (6) growth of real GDP per capita, (7) short term rate (Ordinary funds), and (8) long term rate.

\begin{tabular}{|c|c|c|c|c|c|c|c|c|}
\hline & \multirow[b]{2}{*}{$\begin{array}{c}\text { Buy-Sell } \\
\text { small }\end{array}$} & \multirow[b]{2}{*}{$\begin{array}{c}\text { Buy-Sell } \\
\text { med }\end{array}$} & \multicolumn{3}{|c|}{ Avg. } & \multicolumn{3}{|c|}{ Short } \\
\hline & & & $\begin{array}{c}\text { Buy-Sell } \\
\text { large }\end{array}$ & $\begin{array}{c}\text { cross-sect } \\
\text { monthly } \\
\text { s.d. }\end{array}$ & $\begin{array}{l}\text { Excess } \\
\text { Return }\end{array}$ & $\begin{array}{l}\text { Growth } \\
\mathrm{rgdp} / \mathrm{pc}\end{array}$ & $\begin{array}{c}\text { Term rate } \\
\text { Ordinary } \\
\text { funds } \\
\end{array}$ & $\begin{array}{l}\text { Long } \\
\text { term } \\
\text { rate } \\
\end{array}$ \\
\hline Buy-Sell small & 1 & & & & & & & \\
\hline Buy-Sell med & 0.02 & 1 & & & & & & \\
\hline Buy-Sell large & 0.14 & 0.1 & 1 & & & & & \\
\hline Avg. cross-sect monthly s.d. & 0.17 & 0.12 & 0.15 & 1 & & & & \\
\hline Excess Return & -0.33 & -0.24 & 0.08 & 0.09 & 1 & & & \\
\hline Growth $\mathrm{rgdp} / \mathrm{pc}$ & -0.28 & -0.21 & -0.19 & -0.15 & 0.33 & 1 & & \\
\hline Short term rate Ordinary funds & 0.16 & 0 & 0.13 & -0.23 & -0.41 & -0.03 & 1 & \\
\hline Long term rate & -0.35 & 0.36 & 0.1 & -0.05 & 0.02 & -0.06 & 0.33 & 1 \\
\hline
\end{tabular}




\section{Table 13: Autocorrelation Estimates}

We estimate the autocorrelations of momentum profits for the Victorian and CRSP eras. The T-stat column represents the tstatistics of the estimate under the null that all autocorrelations are zero.

\begin{tabular}{|c|c|c|c|c|}
\hline & \multicolumn{2}{|c|}{ Victorian Era } & \multicolumn{2}{|c|}{ CRSP Era } \\
\hline & Estimate & t-stat & Estimate & t-stat \\
\hline \multicolumn{5}{|c|}{ Panel A : Monthly Data } \\
\hline$\rho_{1}$ & -0.0534 & -1.2410 & 0.0755 & 2.3697 \\
\hline$\rho_{2}$ & -0.1217 & -2.8282 & -0.0919 & -2.8832 \\
\hline$\rho_{3}$ & -0.0477 & -1.1081 & -0.1019 & -3.1959 \\
\hline$\rho_{4}$ & -0.0429 & -0.9970 & -0.0174 & -0.5453 \\
\hline$\rho_{5}$ & 0.0092 & 0.2133 & -0.0191 & -0.5991 \\
\hline$\rho_{6}$ & 0.0095 & 0.2218 & 0.0944 & 2.9619 \\
\hline \multicolumn{5}{|c|}{ Panel B : Quarterly Data } \\
\hline$\rho_{1}$ & -0.1715 & -2.3004 & -0.1119 & -2.0264 \\
\hline$\rho_{2}$ & 0.1550 & 2.0791 & 0.0805 & 1.4576 \\
\hline$\rho_{3}$ & -0.0733 & -1.0373 & -0.0892 & -1.6163 \\
\hline$\rho_{4}$ & 0.1140 & 1.5293 & 0.0410 & 0.7424 \\
\hline
\end{tabular}

\section{Table 14: Tests on the Autocorrelation Feature}

Each entry is p-value. Ljung-Box test is the test on the null that all autocorrelations are zero. We can reject the null except for the monthly data of the Victorian era. Box-Pierce test is the test on the null that autocorrelations are same for both Victorian and CRSP eras. P-values show that we cannot reject the null for both monthly and quarterly data.

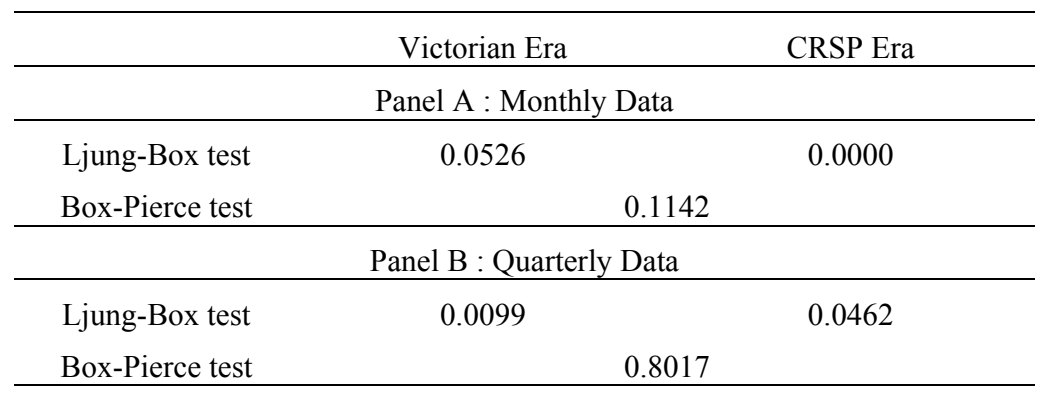




\section{Table 15: Empirical Distribution of Duration Up and Down Momentum Cycles}

Entries feature a comparison of up and down momentum duration cycles for the CRSP and Victorian eras. Entries feature a comparison of up and down momentum duration cycles for the CRSP and Victorian eras. All cycles are computed from the returns of the Lag-6, Hold-6 WML momentum portfolio. A duration spell is a period of consecutive gains or losses. An Up cycle begins when a positive return follows a negative return and continues until the next negative return. A down cycle begins when a negative return follows a positive return and continues until the next positive return.

\begin{tabular}{ccc}
\hline \multicolumn{3}{c}{ Panel A : Up Cycles } \\
\hline Duration Spells & Victorian Era & CRSP Era \\
\hline 1 & 0.35 & 0.33 \\
2 & 0.26 & 0.22 \\
3 & 0.16 & 0.19 \\
4 & 0.09 & 0.10 \\
5 & 0.08 & 0.08 \\
6 and more & 0.06 & 0.08 \\
\hline & Panel B : Down Cycles & \\
\hline Duration Spells & Victorian Era & CRSP Era \\
\hline 1 & 0.61 & 0.58 \\
2 & 0.27 & 0.26 \\
3 & 0.05 & 0.07 \\
4 & 0.05 & 0.06 \\
5 & 0.02 & 0.02 \\
6 and more & 0.00 & 0.00 \\
\hline
\end{tabular}




\section{Table 16: Nonparametric Tests of Duration Spells}

Duration spells of up and down momentum cycles are tested for equality across the Victorian and CRSP eras. The test statistics are: are (a) the Mann-Whitney-Wilcoxon test, (b) Kolmogorov-Smirnov test, (c) Cramer-von Mises test, and (d) Kuiper test Entries to the table are p-values for the null hypothesis that the duration spells during the Victorian and CRSP eras are drawn from the same population.

\begin{tabular}{lccccc}
\hline \multicolumn{1}{c}{ Panel A : Up Cycles } & & & \\
\hline \multicolumn{1}{c}{ Duration Spells } & 1 & 2 & 3 & 4 & 6 \\
\hline Mann-Whitney-Wilcoxon & 0.30 & 0.19 & 0.05 & 0.13 & 0.00 \\
Kolmogorov-Smirnov & 0.90 & 0.79 & 0.31 & 0.34 & 0.08 \\
Cramer-von Mises & 0.16 & 0.19 & 0.41 & 0.13 & 0.94 \\
Kuiper & 0.99 & 0.99 & 0.86 & 0.64 & 0.32 \\
\hline \multicolumn{1}{c}{ Dunation Spells } & 1 & 2 & 3 & 4 & 6 \\
\hline Mann-Whitney-Wilcoxon & 0.30 & 0.08 & 0.09 & 0.12 & 0.57 \\
Kolmogorov-Smirnov & 0.70 & 0.48 & 0.14 & 0.87 & 0.92 \\
Cramer-von Mises & 0.23 & 0.49 & 0.16 & 0.18 & 0.10 \\
Kuiper & 0.82 & 0.94 & 0.41 & 0.94 & 0.92 \\
\hline
\end{tabular}




\section{Table 17: Duration Spells - Parametric Estimates}

We estimate parameters of Exponential distribution, Gamma distribution and Weibull distributions using Maximum Likelihood. The density function of the exponential distribution is $f(t)=\lambda e^{-\lambda t}$, the Gamma distribution is $f(t)=\frac{(t \lambda)^{\kappa-1} \lambda e^{-\lambda t}}{\Gamma(\kappa)}$, and that of the Weibull distribution is $f(t)=\lambda p(\lambda t)^{p-1} e^{-(\lambda t)^{p}}$.

\begin{tabular}{|c|c|c|c|c|c|}
\hline \multirow{2}{*}{$\begin{array}{l}\text { Distributional } \\
\text { Assumption }\end{array}$} & \multirow{2}{*}{ Parameters } & \multicolumn{2}{|c|}{ Victorian Era } & \multicolumn{2}{|c|}{ CRSP Era } \\
\hline & & MLE & t-stat & MLE & t-stat \\
\hline \multicolumn{6}{|c|}{ Panel A : Up Cycles } \\
\hline Exponential & $\lambda$ & 0.4030 & 11.5143 & 0.3696 & 14.9636 \\
\hline \multirow{2}{*}{ Gamma } & $\lambda$ & 1.0985 & 7.8241 & 0.9352 & 10.1652 \\
\hline & $\kappa$ & 2.7255 & 8.5870 & 2.5301 & 11.2349 \\
\hline \multirow{2}{*}{ Weibull } & $\lambda$ & 0.3571 & 18.2194 & 0.3290 & 22.3810 \\
\hline & $\mathrm{p}$ & 1.6730 & 15.3768 & 1.5860 & 20.3856 \\
\hline \multicolumn{6}{|c|}{ Panel B : Down Cycles } \\
\hline Exponential & $\lambda$ & 0.6346 & 11.4964 & 0.5958 & 14.9698 \\
\hline \multirow{2}{*}{ Gamma } & $\lambda$ & 2.7447 & 7.9441 & 2.2371 & 10.3522 \\
\hline & $\kappa$ & 4.3250 & 8.4275 & 3.7551 & 11.0737 \\
\hline \multirow{2}{*}{ Weibull } & $\lambda$ & 0.5583 & 20.6778 & 0.5247 & 25.2260 \\
\hline & $\mathrm{p}$ & 1.9140 & 16.4433 & 1.7985 & 21.3345 \\
\hline
\end{tabular}




\section{Table 18: The Effect of State Variables on the Duration Spells}

We test the effect of state variables on the duration spells. First, we apply 3 month moving average filter to all variables first. Then, remove durations which are less than or equal to 3 . With those filtered data, we test the effect of state variables on the duration spells. We set $\lambda=\exp \left(-\beta_{0}-\beta_{1} r_{f}-\beta_{2} r_{\text {stock }}\right)$. We use $r_{f}$ as the average risk free rate minus the inflation rate of the last year of the beginning of the duration, assuming the inflation rate of last year is the expected inflation rate. $r_{\text {stock }}$ is the average stock market return for the last three years. As predicted the point estimate on the risk-free rate has a positive effect on the duration of up cycles and a negative effect on the duration of down cycles. Regardless of specification the point estimates are always the predicted sign but often insignificant from zero.

\begin{tabular}{|c|c|c|c|c|c|c|c|}
\hline \multirow{2}{*}{$\begin{array}{l}\text { Distributional } \\
\text { Assumption }\end{array}$} & \multirow{2}{*}{ Parameters } & \multicolumn{3}{|c|}{ Victorian Era } & \multicolumn{3}{|c|}{ CRSP Era } \\
\hline & & MLE & s.e. & t-stat & MLE & s.e. & t-stat \\
\hline \multicolumn{8}{|c|}{ Panel A : Up Cycles } \\
\hline \multirow{4}{*}{ Gamma } & $\beta_{0}$ & 0.2923 & 0.3252 & 0.8988 & 0.2004 & 0.1878 & 1.0671 \\
\hline & $\beta_{1}$ & 40.2349 & 47.1920 & 0.8526 & 36.2294 & 17.6612 & 2.0514 \\
\hline & $\beta_{2}$ & -0.2851 & 0.6823 & -0.4179 & -0.0419 & 0.1499 & -0.2795 \\
\hline & $\kappa$ & 5.6320 & 1.3275 & 4.2426 & 6.0021 & 1.0096 & 5.9450 \\
\hline \multirow{4}{*}{ Weibull } & $\beta_{0}$ & 2.1146 & 0.2337 & 9.0484 & 2.1174 & 0.0926 & 22.8661 \\
\hline & $\beta_{1}$ & 75.7509 & 43.3978 & 1.7455 & 34.1785 & 20.0536 & 1.7044 \\
\hline & $\beta_{2}$ & -0.4543 & 0.7842 & -0.5793 & -0.0427 & 0.1692 & -0.2524 \\
\hline & $\mathrm{p}$ & 2.3417 & 0.2946 & 7.9487 & 2.3697 & 0.2059 & 11.5090 \\
\hline \multicolumn{8}{|c|}{ Panel B : Down Cycles } \\
\hline \multirow{4}{*}{ Gamma } & $\beta_{0}$ & -0.4157 & 0.5012 & -0.8294 & -0.4929 & 0.2688 & -1.8337 \\
\hline & $\beta_{1}$ & -101.0582 & 59.7824 & -1.6904 & -19.1313 & 21.7347 & -0.8802 \\
\hline & $\beta_{2}$ & -1.3545 & 0.6565 & -2.0632 & 0.0322 & 0.1378 & 0.2337 \\
\hline & $\kappa$ & 16.1790 & 6.3200 & 2.5600 & 9.2884 & 2.3967 & 3.8755 \\
\hline \multirow{4}{*}{ Weibull } & $\beta_{0}$ & 2.7569 & 0.3105 & 8.8789 & 1.8576 & 0.0811 & 22.9051 \\
\hline & $\beta_{1}$ & -145.4752 & 55.6524 & -2.6140 & -33.0879 & 24.6850 & -1.3404 \\
\hline & $\beta_{2}$ & -1.9172 & 0.6154 & -3.1154 & 0.0320 & 0.1478 & 0.2165 \\
\hline & $\mathrm{p}$ & 4.4620 & 0.9786 & 4.5596 & 2.9876 & 0.4095 & 7.2957 \\
\hline
\end{tabular}


Table 19: The Difference of the Effect of State Variables on the Duration Spells across up/down Cycles

We use the same smoothing as in Table 18. The null hypothesis is that the level of the risk-free rate differs across UP and DOWN cycles. We test this hypothesis by pooling the UP and Down cycles and estimating allowing the all coefficients to vary with an interaction dummy equal to one if the observation is a down cycle. Formally, we set $\lambda=$ $\exp \left(-\beta_{0}-\beta_{1} r_{f}-\beta_{2} r_{\text {stock }}+\right.$ down dummy $\left.\left(-\beta_{0, d d}-\beta_{1, d d} r_{f}-\beta_{2, d d} r_{\text {stock }}\right)\right)$ where down dummy is 1 if the cycle is down or 0 otherwise. In the same way, $\kappa$ and $\mathrm{p}$ are adjusted. Regardless of era or specification, the effect of the risk-free rate is always significantly lower in DOWN cycles.

\begin{tabular}{|c|c|c|c|c|c|c|c|}
\hline \multirow{2}{*}{$\begin{array}{l}\text { Distributional } \\
\text { Assumption }\end{array}$} & \multirow{2}{*}{ Parameters } & \multicolumn{3}{|c|}{ Victorian Era } & \multicolumn{3}{|c|}{ CRSP Era } \\
\hline & & MLE & s.e. & t-stat & MLE & s.e. & t-stat \\
\hline \multirow{8}{*}{ Gamma } & $\beta_{0}$ & 0.2923 & 0.3208 & 0.9112 & 0.2004 & 0.1878 & 1.0671 \\
\hline & $\beta_{0, d d}$ & -0.7079 & 0.5815 & -1.2174 & -0.6933 & 0.3278 & -2.1150 \\
\hline & $\beta_{1}$ & 40.2349 & 41.2217 & 0.9761 & 36.2294 & 18.7357 & 1.9337 \\
\hline & $\beta_{1, d d}$ & -141.293 & 63.6805 & -2.2188 & -55.3607 & 28.1399 & -1.9673 \\
\hline & $\beta_{2}$ & -0.2851 & 0.6822 & -0.4179 & -0.0419 & 0.1499 & -0.2795 \\
\hline & $\beta_{2, d d}$ & -1.0694 & 0.9387 & -1.1392 & 0.0741 & 0.2035 & 0.3641 \\
\hline & $\kappa$ & 5.632 & 1.3274 & 4.2429 & 6.0021 & 1.0096 & 5.9450 \\
\hline & $\kappa \_d d$ & 10.547 & 6.3601 & 1.6583 & 3.2862 & 2.5999 & 1.2640 \\
\hline \multirow{8}{*}{ Weibull } & $\beta_{0}$ & 2.1146 & 0.234 & 9.0368 & 2.1174 & 0.0926 & 22.8661 \\
\hline & $\beta_{0, d d}$ & 0.6423 & 0.3771 & 1.7033 & -0.2598 & 0.1231 & -2.1105 \\
\hline & $\beta_{1}$ & 75.751 & 43.5596 & 1.7390 & 34.1784 & 20.6532 & 1.6549 \\
\hline & $\beta_{1, d d}$ & -221.2262 & 67.9522 & -3.2556 & -67.2663 & 31.596 & -2.1289 \\
\hline & $\beta_{2}$ & -0.4543 & 0.7839 & -0.5795 & -0.0427 & 0.1692 & -0.2524 \\
\hline & $\beta_{2, d d}$ & -1.4629 & 0.9843 & -1.4862 & 0.0747 & 0.2245 & 0.3327 \\
\hline & $\mathrm{p}$ & 2.3417 & 0.2946 & 7.9487 & 2.3697 & 0.2059 & 11.5090 \\
\hline & p_dd & 2.1203 & 1.0187 & 2.0814 & 0.618 & 0.458 & 1.3493 \\
\hline
\end{tabular}




\section{Table 20: Momentum Portfolio Turnover: Transition Probability Matrix}

The turnover of the relative strength portfolios is measured via a transition probability matrix for the $\mathrm{K}=6 / \mathrm{J}=6$ strategy. The diagonal elements are of most interest as they reveal what fraction of stocks remain in the portfolio.

Portfolio at time $\mathrm{t}$

Probability of being in

Portfolio at time $\mathrm{t}+6$

\begin{tabular}{llcccccccccc}
\hline \multirow{2}{*}{ Size } & & Small & Med & Large & Small & Med & Large & Small & Med & Large \\
\cline { 3 - 10 } & & & & & & & & & & \\
\multirow{2}{*}{ Small } & Sell & 0.345 & 0.051 & 0.000 & 0.282 & 0.023 & 0.000 & 0.322 & 0.033 & 0.000 \\
Med & Sell & 0.008 & 0.296 & 0.032 & 0.008 & 0.207 & 0.014 & 0.010 & 0.232 & 0.019 \\
Large & Sell & 0.000 & 0.009 & 0.306 & 0.001 & 0.007 & 0.211 & 0.000 & 0.008 & 0.243 \\
Small & Med & 0.202 & 0.008 & 0.000 & 0.281 & 0.009 & 0.000 & 0.212 & 0.009 & 0.000 \\
Med & Med & 0.011 & 0.224 & 0.009 & 0.018 & 0.354 & 0.012 & 0.013 & 0.276 & 0.012 \\
Large & Med & 0.000 & 0.017 & 0.310 & 0.000 & 0.026 & 0.452 & 0.000 & 0.014 & 0.336 \\
Small & Buy & 0.282 & 0.006 & 0.000 & 0.256 & 0.005 & 0.000 & 0.301 & 0.005 & 0.000 \\
Med & Buy & 0.039 & 0.279 & 0.005 & 0.036 & 0.263 & 0.005 & 0.045 & 0.308 & 0.006 \\
Large & Buy & 0.000 & 0.031 & 0.279 & 0.000 & 0.027 & 0.246 & 0.000 & 0.035 & 0.321 \\
No Price at t+6 & 0.113 & 0.079 & 0.058 & 0.119 & 0.079 & 0.059 & 0.096 & 0.078 & 0.062 \\
\hline
\end{tabular}




\section{Table 21: Bid-Ask Spread Statistics}

The relative strength portfolios are formed on a 6-month lagged returns and held for 6 months. The stocks are ranked in ascending order on the basis of 6-month lagged returns. A value-weighted portfolio of stocks in the lowest 33rd percentile past return is the sell portfolio. A value-weighted portfolio of stocks in the highest 67 th percentile past return is the buy portfolio. Value weighted average of the Bid-Ask spread of each security that is added or removed from the portfolio at time $t$, where Bid-Ask Spread $=($ AskBid)/[average(bid,ask)]

\begin{tabular}{|c|c|c|c|c|c|c|c|c|}
\hline \multicolumn{3}{|c|}{ Sell } & \multicolumn{3}{|c|}{ Mid } & \multicolumn{3}{|c|}{ Buy } \\
\hline Small & Med & Large & Small & Med & Large & Small & Med & Large \\
\hline \multicolumn{9}{|c|}{ Prob of remaining in portfolio } \\
\hline 0.345 & 0.296 & 0.306 & 0.281 & 0.354 & 0.452 & 0.301 & 0.308 & 0.321 \\
\hline \multicolumn{9}{|c|}{ Bid-ask spread of those traded } \\
\hline 0.21 & 0.09 & 0.03 & 0.14 & 0.06 & 0.02 & 0.15 & 0.07 & 0.02 \\
\hline \multicolumn{9}{|c|}{ Percentage of "new" stocks in new portfolio } \\
\hline 0.655 & 0.704 & 0.694 & 0.719 & 0.646 & 0.548 & 0.699 & 0.692 & 0.679 \\
\hline \multicolumn{9}{|c|}{ Per period Bid-Ask cost trading cost } \\
\hline 0.0229 & 0.0106 & 0.0035 & 0.0168 & 0.0065 & 0.0018 & 0.0175 & 0.0081 & 0.0023 \\
\hline \multicolumn{9}{|c|}{ Per period costs in basis points } \\
\hline 229 & 106 & 35 & 168 & 65 & 18 & 175 & 81 & 23 \\
\hline
\end{tabular}




\section{Figure 1: Momentum Profit Cycles}

The figure displays the time series of the Fama-French momentum factor returns from 1946 to 2008 and the momentum factor returns during the Victorian era.

Fama-French Momentum Returns

Centered 1-Year Moving Average: 1946-2008

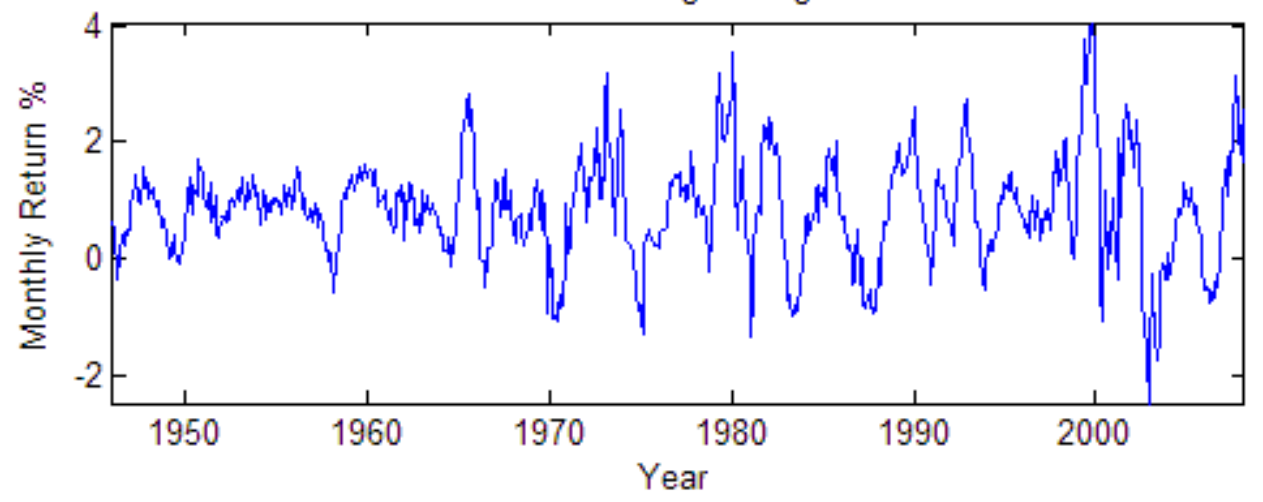

Momentum Portfolio Returns

Centered 1-Year Moving Average: 1867-1907

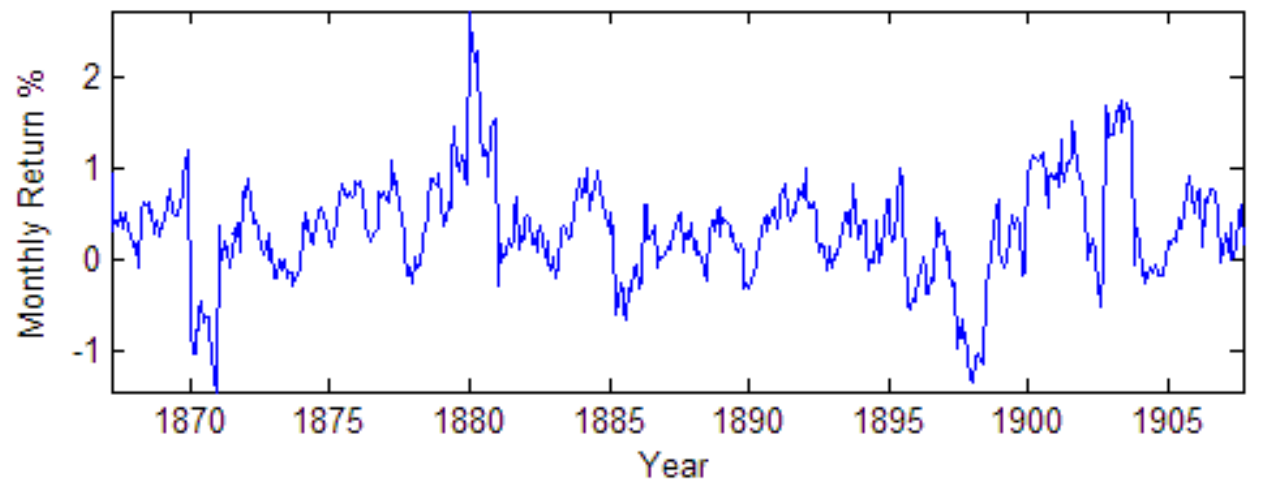




\section{Figure 2: Number of Stocks in Portfolios}

This study makes use of a new data set of 1,808 stocks (equity) listed in London between 1866 and 1907 . The plots display the number of stocks, which decline to 985 in 1903 and 544 in 1904 - due to a number of industries vanishing from the quotation list, only to reappear in 1905.

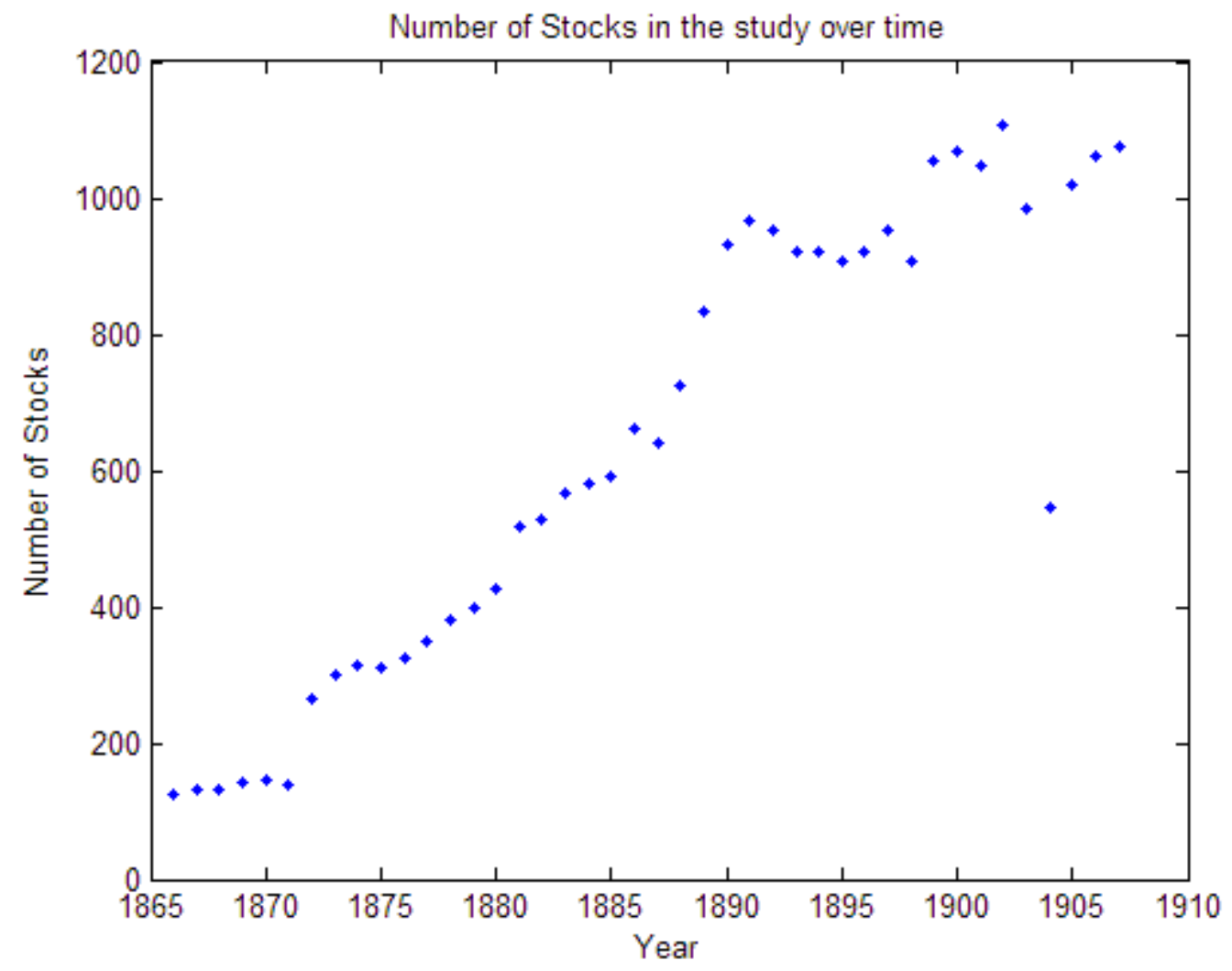




\section{Figure 3: Five-Year Rolling Average Returns on Size-based Relative Strength Portfolios}

We report the average monthly excess return on the momentum portfolios during each of the five years following portfolio formation, after skipping a month. The relative strength portfolios are formed on a 6-month lagged return and held for 6 months. The stocks are ranked in ascending order on the basis of 6-month lagged returns. A value-weighted portfolio of stocks in the lowest 33rd percentile past return is the sell portfolio. A value-weighted portfolio of stocks in the highest 67 th percentile past return is the buy portfolio.

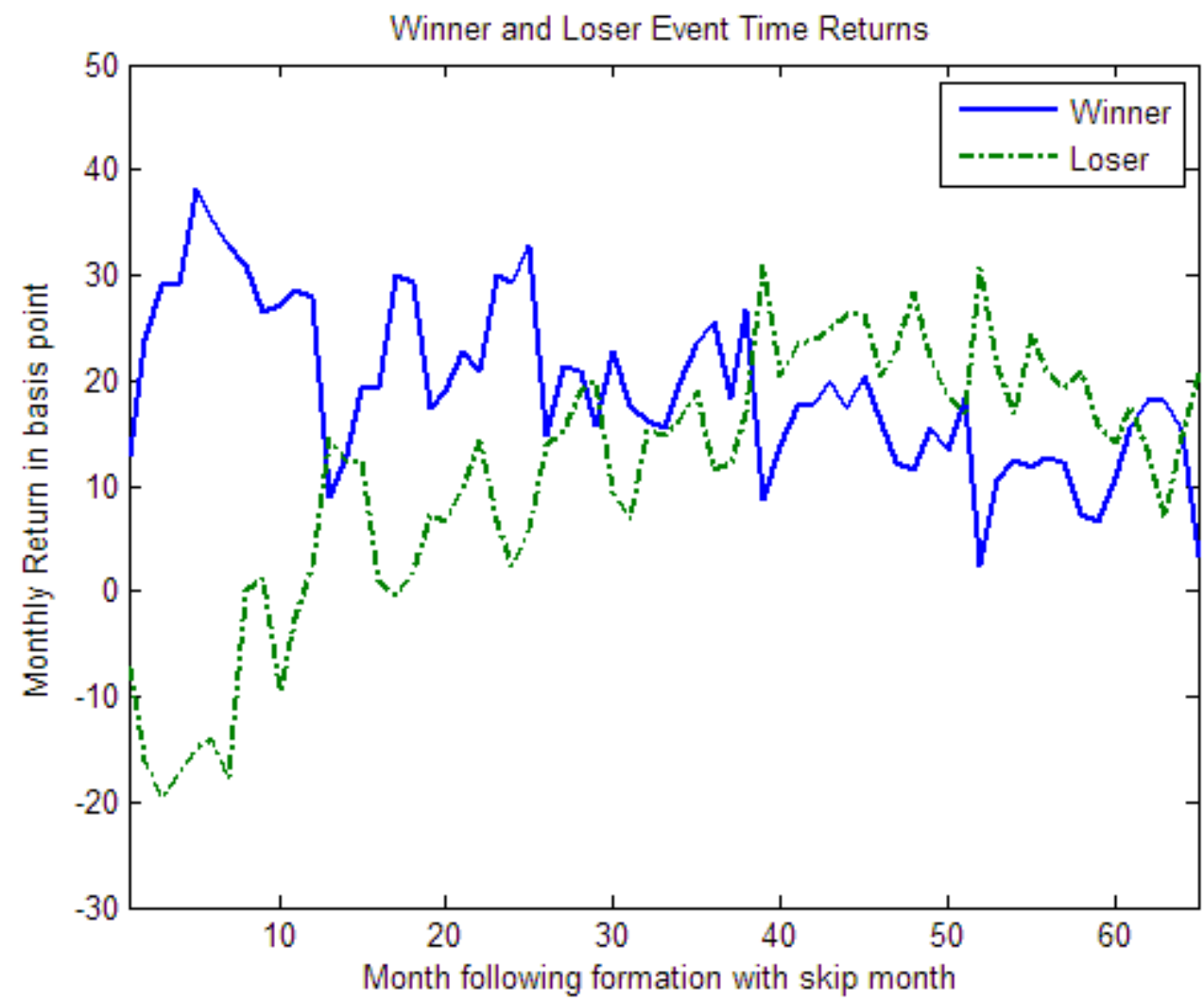


Figure 4: Winner and Looser Portfolio Investment

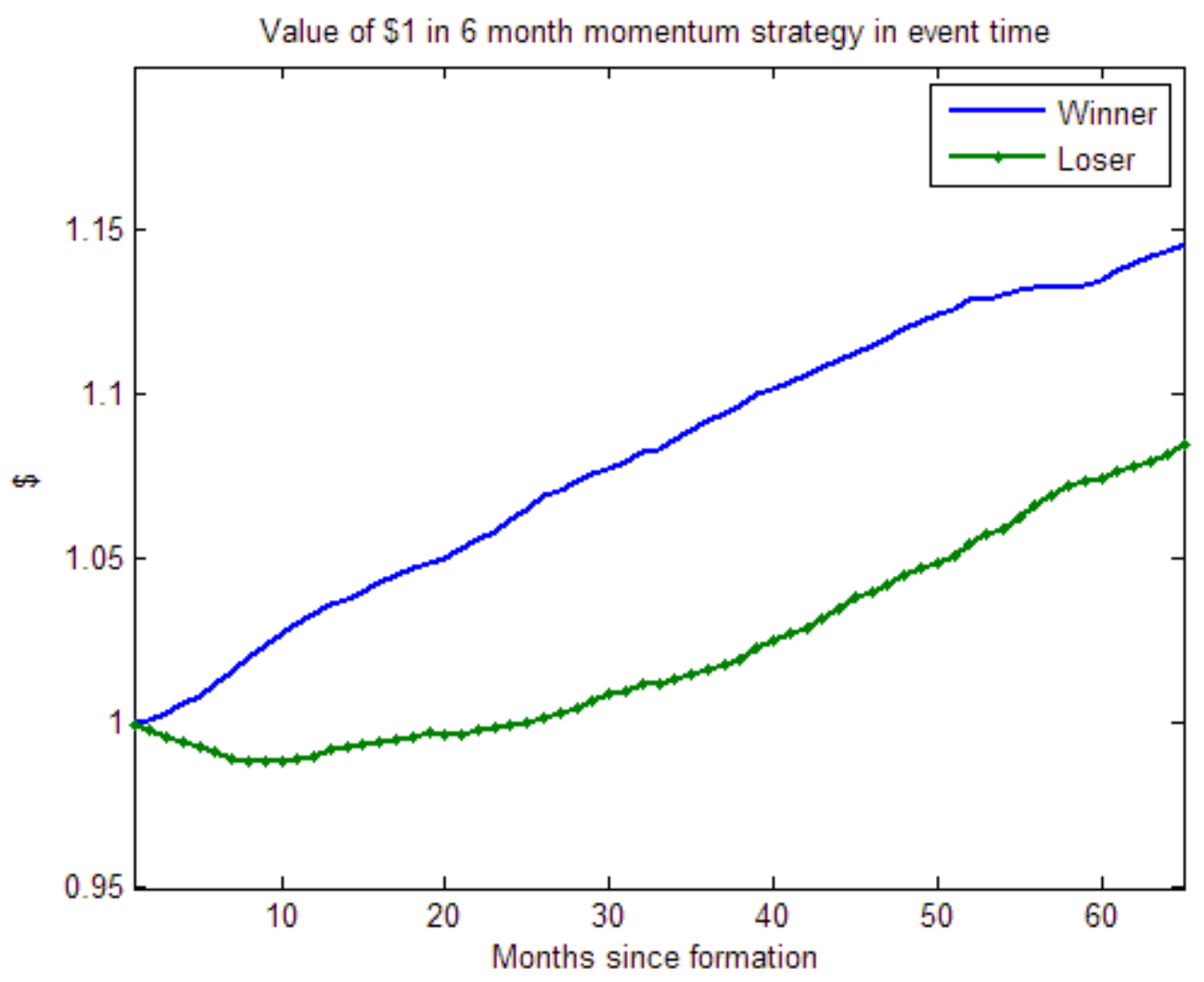


Figure 5: Winner Minus Looser Investment

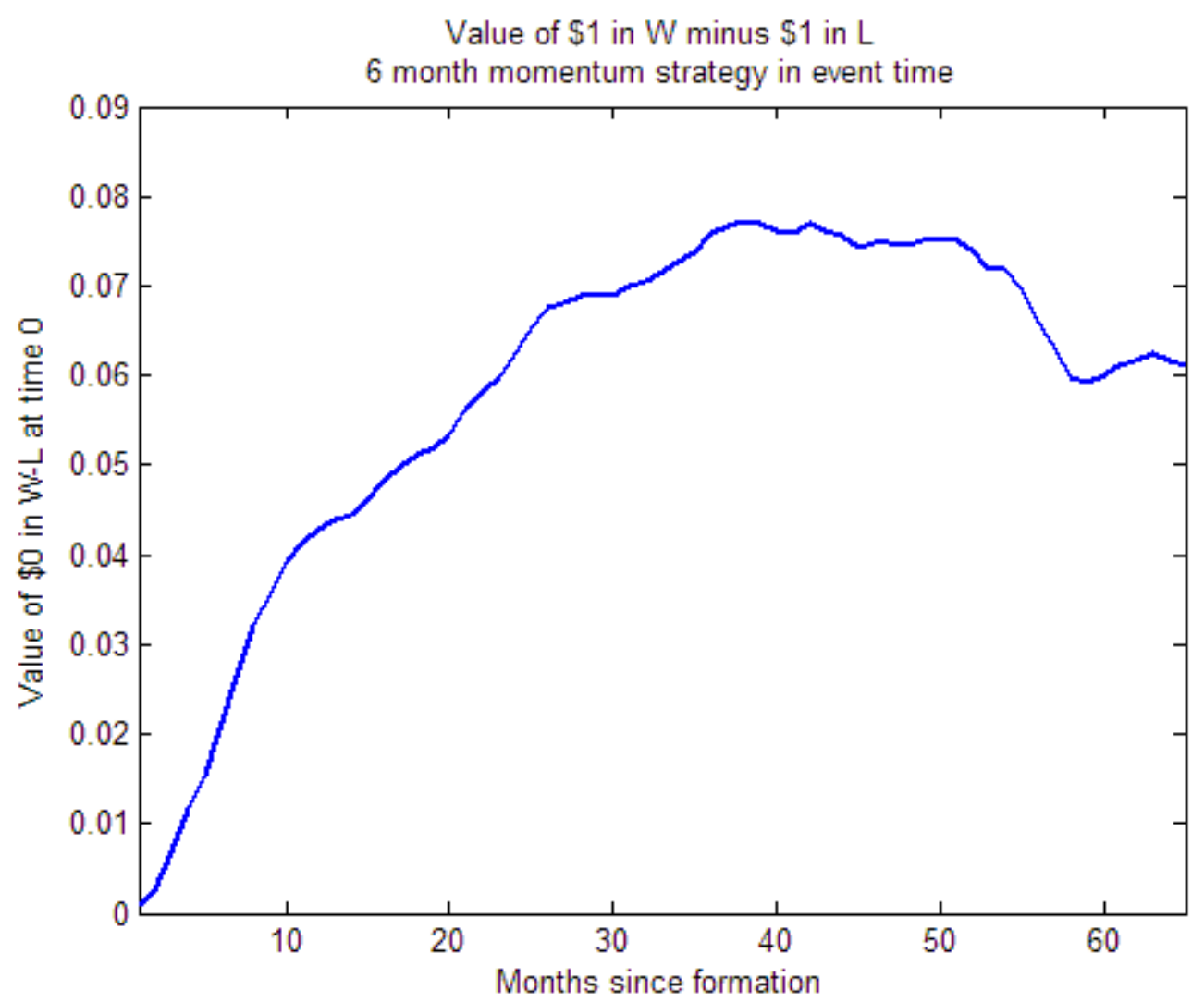


Figure 6: Empirical and Parametric distribution of duration up
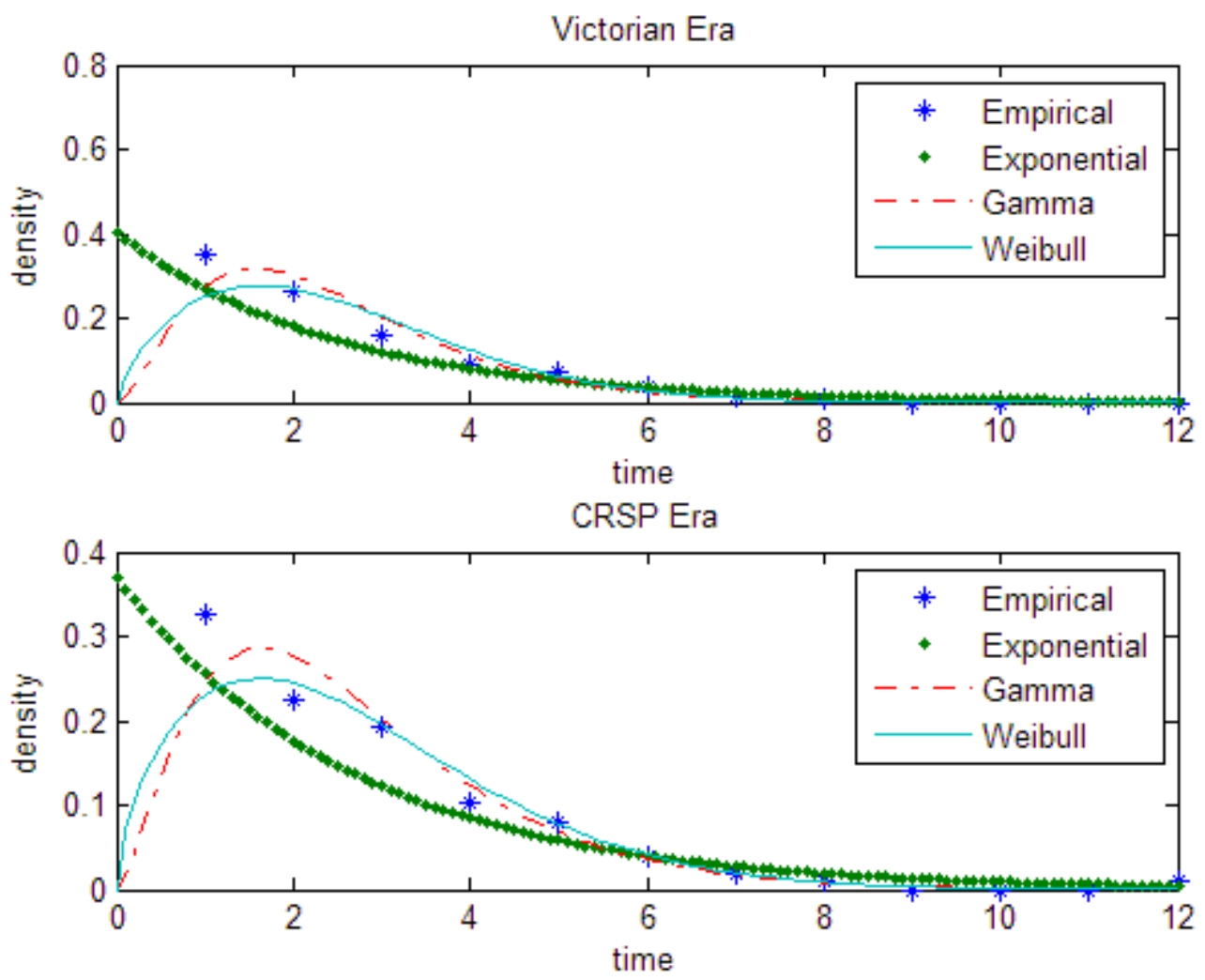
Figure 7: Empirical and Parametric distribution of duration down
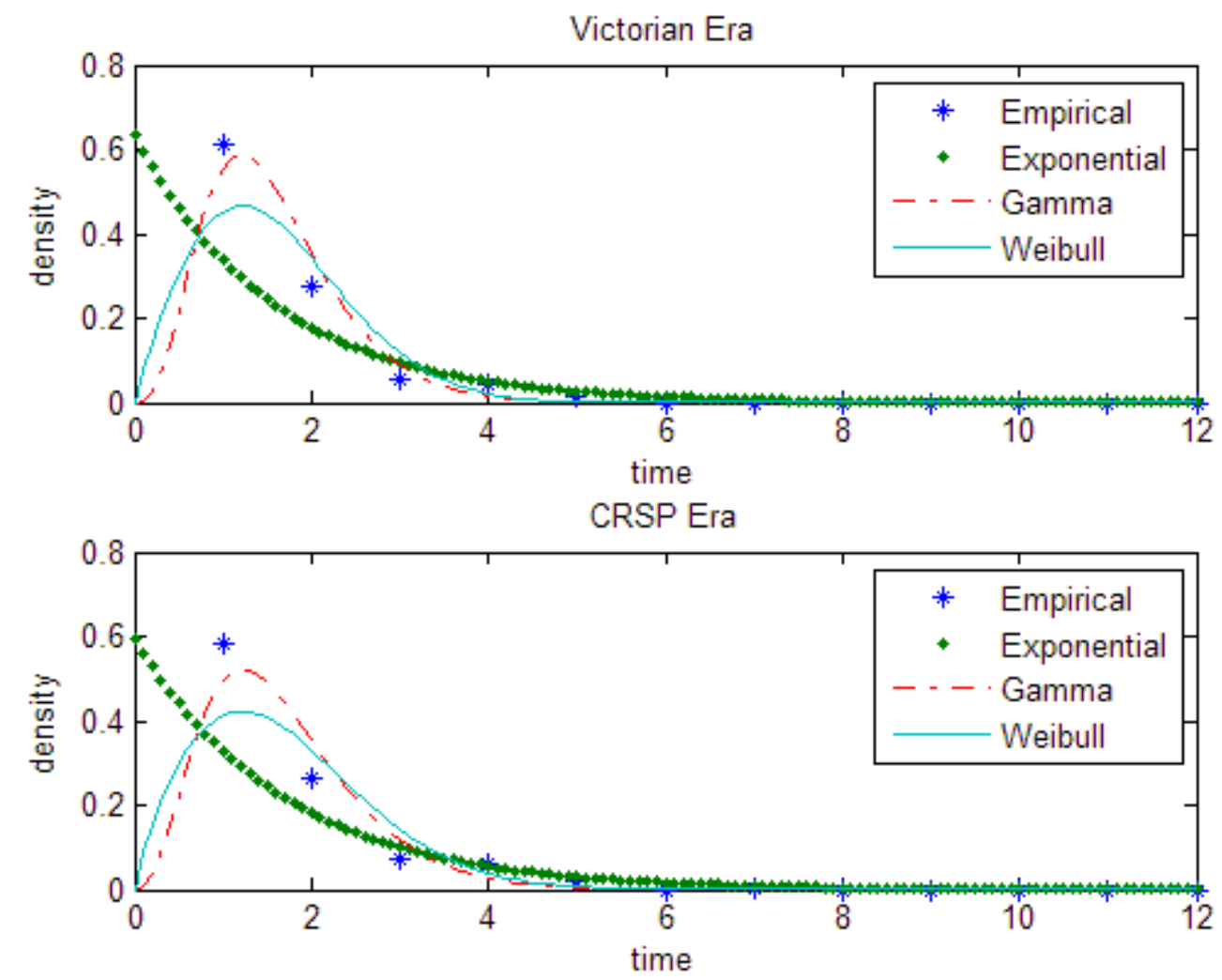